\title{
A Central Amygdala-Globus Pallidus Circuit Conveys Unconditioned Stimulus-Related Information and Controls Fear Learning
}

\author{
${ }^{\circ}$ Jacqueline Giovanniello, ${ }^{1,2}{ }^{\circledR}$ Kai Yu, ${ }^{2 \star}$ Alessandro Furlan, ${ }^{2 \star}$ Gregory Thomas Nachtrab, ${ }^{3}$ Radhashree Sharma, ${ }^{2}$ \\ Xiaoke Chen, ${ }^{3}$ and ${ }^{\circledR} \mathrm{Bo} \mathrm{Li}^{1,2}$ \\ ${ }^{1}$ School of Biological Sciences, Cold Spring Harbor Laboratory, Cold Spring Harbor, New York 11724, ${ }^{2}$ Cold Spring Harbor Laboratory, Cold Spring \\ Harbor, New York 11724, and ${ }^{3}$ Department of Biology, Stanford University, California 94305
}

The central amygdala (CeA) is critically involved in a range of adaptive behaviors, including defensive behaviors. Neurons in the CeA send long-range projections to a number of extra-amygdala targets, but the functions of these projections remain elusive. Here, we report that a previously neglected CeA-to-globus pallidus external segment (GPe) circuit plays an essential role in classical fear conditioning. By anatomic tracing, in situ hybridization and channelrhodopsin (ChR2)-assisted circuit mapping in both male and female mice, we found that a subset of CeA neurons send projections to the GPe, and the majority of these GPe-projecting CeA neurons express the neuropeptide somatostatin. Notably, chronic inhibition of GPe-projecting CeA neurons with the tetanus toxin light chain (TeLC) completely blocks auditory fear conditioning. In vivo fiber photometry revealed that these neurons are selectively excited by the unconditioned stimulus (US) during fear conditioning. Furthermore, transient optogenetic inactivation or activation of these neurons selectively during US presentation impairs or promotes, respectively, fear learning. Our results suggest that a major function of GPe-projecting CeA neurons is to represent and convey US-related information through the CeA-GPe circuit, thereby regulating learning in fear conditioning.

Key words: central amygdala; fear conditioning; globus pallidus; learning; somatostatin; unconditioned stimulus

\section{Significance Statement}

The central amygdala (CeA) has been implicated in the establishment of defensive behaviors toward threats, but the underlying circuit mechanisms remain unclear. Here, we found that a subpopulation of neurons in the CeA, which are mainly those that express the neuropeptide somatostatin, send projections to the globus pallidus external segment (GPe), and this CeA-GPe circuit conveys unconditioned stimulus (US)-related information during classical fear conditioning, thereby having an indispensable role in learning. Our results reveal a previously unknown circuit mechanism for fear learning.

Received Aug. 8, 2020; revised 0ct. 4, 2020; accepted Oct. 12, 2020

Author contributions: J.G. and B.L. designed research; J.G., K.Y., A.F., and R.S. performed research; G.T.N.

and X.C. contributed unpublished reagents/analytic tools; J.G. analyzed data; B.L. wrote the paper.

*K.Y. and A.F. contributed equally to this work.

This work was supported by the EMBO Grant ALTF 458-2017 (to A.F.); the Swedish Research Council Grant 2017-00333 (to A.F.); Charles H. Revson Senior Fellowships in Biomedical Science (A.F.); National Institutes of Health Grants R01MH101214, R01MH108924, and R01NS104944 (to B.L.); the Human Frontier Science Program Grant RGP0015/2016 (to B.L.); the Stanley Family Foundation (B.L.); the Simons Foundation Grant 344904 (to B.L.); the Wodecroft Foundation (B.L.), the Cold Spring Harbor Laboratory and Northwell Health Affiliation (B.L.); and the Feil Family Neuroscience Endowment (B.L.). We thank members of the Li laboratory for helpful discussions and Z. Josh Huang for providing the H2B-GFP (Rosa26-stop ${ }^{\text {flox }}$-H2B-GFP) reporter mice.

J. Giovanniello's present address: Department of Psychology, University of California, Los Angeles, California 90095

The authors declare no competing financial interests.

Correspondence should be addressed to Bo Li at bli@cshl.edu.

https://doi.org/10.1523/JNEUROSCI.2090-20.2020

Copyright $\odot 2020$ the authors

\section{Introduction}

The central amygdala (CeA) plays important roles in learning and executing adaptive behaviors. In particular, its function in the acquisition and expression of defensive behaviors has received arguably the most intensive study (Duvarci and Pare, 2014; Herry and Johansen, 2014; Janak and Tye, 2015). For example, transient pharmacological inactivation of the CeA (Goosens and Maren, 2003; Wilensky et al., 2006), or specific inactivation of the lateral division of the CeA (CeL; Ciocchi et al., 2010), during Pavlovian fear conditioning blocks the formation of fear memories. Moreover, in vivo single unit recording demonstrates that fear conditioning causes increased spiking in one CeA population (the "ON" neurons) and decreased spiking in another (the "OFF" neurons) in response to cues predicting shocks. Such learning-induced changes in the responsiveness of CeA neurons to CS presentations may facilitate the expression of learned defensive responses, including conditioned freezing behavior (Ciocchi et al., 2010; Haubensak et al., 2010; Duvarci et 
al., 2011). These findings have led to the notion that the CeA is essential for the formation of aversive memories.

The CeA is a striatal-like structure that contains medium spiny neurons mainly derived from the lateral ganglionic eminence during development (Swanson and Petrovich, 1998; Cassell et al., 1999; García-López et al., 2008; Waraczynski, 2016). These neurons show considerable heterogeneity (Fadok et al., 2018; Li, 2019), which is partly revealed by the different genetic or neurochemical markers that these neurons express. Two of these markers, somatostatin (Sst; Cassell and Gray, 1989) and protein kinase C- $\delta$ (PKC- $\delta$; Haubensak et al., 2010), label two major populations that are largely nonoverlapping in the CeA (Haubensak et al., 2010; Li et al., 2013; Li, 2019).

Recent studies have shown that the excitatory synaptic transmission onto Sst-expressing $\left(\mathrm{Sst}^{+}\right) \mathrm{CeA}$ neurons is potentiated, whereas that onto Sst-negative $\left(\mathrm{Sst}^{-}\right) \mathrm{CeA}$ neurons (which are mainly PKC- $\delta^{+}$neurons) is weakened by fear conditioning ( $\mathrm{Li}$ et al., 2013; Penzo et al., 2014, 2015; Ahrens et al., 2018; Hartley et al., 2019). Consistently, in vivo fiber photometry (Yu et al., 2016) or single unit recording (Fadok et al., 2017) studies demonstrate that $\mathrm{Sst}^{+} \mathrm{CeA}$ neurons show increased excitatory responses to shock-predicting cues following fear conditioning, and the responses correlate with freezing behavior (Fadok et al., 2017). Moreover, inhibition of $\mathrm{Sst}^{+}$CeA neurons during fear conditioning using chemogenetic (Li et al., 2013; Penzo et al., 2015), optogenetic (Li et al., 2013) or molecular (Yu et al., 2017) methods, which can abolish the fear conditioning-induced potentiation of excitatory synapses onto these neurons ( $\mathrm{Li}$ et al., 2013; Penzo et al., 2015), impairs the formation of fear memories. These studies provide compelling evidence that $\mathrm{Sst}^{+} \mathrm{CeA}$ neurons constitute an important element of the circuitry underlying fear conditioning.

In light of previous findings about the organization of CeA circuit (Duvarci and Pare, 2014; Herry and Johansen, 2014; Fadok et al., 2018; Li, 2019), Sst $^{+}$CeA neurons can potentially influence fear conditioning via their inhibitory interactions with other neurons locally within the CeA and the resulting disinhibition of the medial division of the CeA (CeM; Ciocchi et al., 2010; Li et al., 2013), which has been shown to control the expression of freezing behavior during fear conditioning through interactions with the midbrain periaqueductal gray (PAG; Krettek and Price, 1978; Veening et al., 1984; LeDoux et al., 1988; Davis, 2000; Duvarci et al., 2011; Tovote et al., 2016; Fadok et al., 2017). Alternatively, or in addition, as $\mathrm{Sst}^{+} \mathrm{CeA}$ neurons also project to many areas outside of the CeA (Penzo et al., 2014; Yu et al., 2017; Ahrens et al., 2018; Fadok et al., 2018; Zhou et al., 2018; Li, 2019; Ye and Veinante, 2019; Steinberg et al., 2020), these neurons may influence fear conditioning through their long-range projections to extra-CeA structures.

Here, we discovered that a subset of CeA neurons send projections to the globus pallidus external segment (GPe), a basal ganglia structure that is best known for its role in motor control (Kita, 2007; Wallace et al., 2017) but has also been implicated in regulating emotions or affects, including fear or threat, in both humans and animals (Blanchard et al., 1981; Hernadi et al., 1997; Baumann et al., 1999; Critchley et al., 2001; Murphy et al., 2003; Talalaenko et al., 2006; Kertes et al., 2009; Sztainberg et al., 2011; Hattingh et al., 2012; Shucard et al., 2012; Ipser et al., 2013; Binelli et al., 2014). Notably, these GPe-projecting CeA neurons are predominantly $\mathrm{Sst}^{+}$neurons. Through in vivo fiber photometry and molecular and optogenetic manipulations, we revealed that this previously neglected CeA-GPe circuit has a critical role in representing information about the unconditioned stimulus (US) and regulating learning during fear conditioning.

\section{Materials and Methods \\ Animals}

Male and female mice of three to six months old were used in the behavioral experiments; those of 6-10 weeks old were used in the in vitro electrophysiology experiments. Mice were housed under a 12/12 h light/ dark cycle (7 A.M. to 7 P.M. light) in groups of two to five animals, with food and water available ad libitum. All behavioral experiments were performed during the light cycle. Littermates were randomly assigned to different groups before experiments. All mice were bred onto a C57BL/ $6 \mathrm{~J}$ background. All experimental procedures were approved by the Institutional Animal Care and Use Committee of Cold Spring Harbor Laboratory (CSHL) and performed in accordance to the US National Institutes of Health guidelines.

The C57/B6 wild-type mice were purchased from the Jackson Laboratory. The H2B-GFP (Rosa26-stop ${ }^{\text {flox }}$-H2B-GFP) reporter mouse line (He et al., 2012) was generated by Z. Josh Huang's lab at CSHL. The Sst-IRES-Cre mice (Taniguchi et al., 2011) were purchased from the Jackson Laboratory (stock \#013044). The Ai14 reporter mice (Madisen et al., 2010) were purchased from the Jackson Laboratory (stock \#007908).

\section{Viral vectors and reagents}

The retrograde AAV expressing Cre (AAVrg-Cre), which is suitable for retrogradely labeling CeA neurons (Keyes et al., 2020), was developed and packed in Xiaoke Chen's lab at Stanford University. The AAV2/9CAG-DIO-TeLC-eGFP was previously described (Murray et al., 2011) and custom-packed at Penn Vector Core. The AAV9-EF1a-DIO-hChR2 (H134R)-eYFP-WPRE-hGH were made by Penn Vector Core. The AAV9-CAG-Flex-GFP was produced by the University of North Carolina vector core facility. The AAV1.Syn.Flex.GCaMP6f.WPRE. SV40, AAV1-hSyn1-SIO-stGtACR1-FusionRed and AAV2-hSyn-DIOmCherry were produced by Addgene. All viral vectors were stored in aliquots at $-80^{\circ} \mathrm{C}$ until use.

The retrograde tracer cholera toxin subunit $\mathrm{B}(\mathrm{CTB})$ conjugated with either Alexa Fluor 647, Alexa Fluor 555, Alexa Fluor or 488 (CTB-647, CTB-555, and CTB-488, respectively) was purchased from Invitrogen, Thermo Fisher Scientific. CTB was used at a concentration of $1 \mathrm{mg} / \mathrm{ml}$ in PBS.

\section{Stereotaxic surgery}

Standard surgical procedures were followed for stereotaxic injection $(\mathrm{Li}$ et al., 2013; Penzo et al., 2015; Yu et al., 2016, 2017). Briefly, mice were anesthetized with isoflurane (3\% at the beginning and $1 \%$ for the rest of the surgical procedure), and positioned in a stereotaxic injection frame (myNeuroLab.com). A digital mouse brain atlas was linked to the injection frame to guide the identification and targeting (Angle Two Stereotaxic System; Leica Biosystems Division of Leica, Buffalo Grove, IL, USA). The injection was performed at the following stereotaxic coordinates for CeL: $-1.22 \mathrm{~mm}$ from bregma, $2.9 \mathrm{~mm}$ lateral from the midline, and $4.6 \mathrm{~mm}$ vertical from skull surface; for GPe: $-0.46 \mathrm{~mm}$ from bregma, $1.85 \mathrm{~mm}$ lateral from the midline, and $3.79 \mathrm{~mm}$ vertical from skull surface; and for BNST: $0.20 \mathrm{~mm}$ from bregma, $0.85 \mathrm{~mm}$ lateral from the midline, and $4.15 \mathrm{~mm}$ vertical from skull surface.

For virus or tracer injection, we made a small cranial window (1-2 $\left.\mathrm{mm}^{2}\right)$, through which virus or fluorescent tracers $(\sim 0.3 \mu \mathrm{l})$ were delivered via a glass micropipette (tip diameter, $\sim 5 \mu \mathrm{m}$ ) by pressure application $(5-20 \mathrm{psi}, 5-20 \mathrm{~ms}$ at $0.5 \mathrm{~Hz})$ controlled by a Picrospritzer III (General Valve) and a pulse generator (Agilent). During the surgical procedure, mice were kept on a heating pad maintained at $35^{\circ} \mathrm{C}$ and were brought back to their home-cage for postsurgery recovery and monitoring. Subcutaneous Metacam (1-2 $\mathrm{mg} \mathrm{kg}^{-1}$ meloxicam; Boehringer Ingelheim Vetmedica) was given postoperatively for analgesia and antiinflammatory purposes. For optogenetic experiments, optical fibers (200- $\mu$ m diameter, $0.22 \mathrm{NA}, 5$-mm length) were implanted bilaterally 0.3 $\mathrm{mm}$ over the CeA. A small metal bar, which was used to hold the mouse 
in the head fixation frame to connect optical fibers during training, was mounted on the skull with $\mathrm{C} \& \mathrm{~B}$ Metabond quick adhesive cement (Parkell Inc.), followed by dental cement (Lang Dental Manufacturing Co).

\section{In vitro electrophysiology}

For the in vitro electrophysiology experiments, mice were anaesthetized with isoflurane and perfused intracardially with $20-\mathrm{ml}$ ice-cold artificial CSF (ACSF; $118 \mathrm{~mm} \mathrm{NaCl}, 2.5 \mathrm{~mm} \mathrm{KCl}, 26.2 \mathrm{~mm} \mathrm{NaHCO}, 1 \mathrm{~mm}$ $\mathrm{NaH}_{2} \mathrm{PO}_{4}, 20 \mathrm{~mm}$ glucose, $2 \mathrm{~mm} \mathrm{MgCl}_{2}$, and $2 \mathrm{~mm} \mathrm{CaCl}_{2}, \mathrm{pH} 7.4$, gassed with $95 \% \mathrm{O}_{2}$ and $5 \% \mathrm{CO}_{2}$ ). Mice were then decapitated and their brains quickly removed and submerged in ice-cold dissection buffer $(110.0 \mathrm{mM}$ choline chloride, $25.0 \mathrm{~mm} \mathrm{NaHCO}_{3}, 1.25 \mathrm{~mm} \mathrm{NaH}_{2} \mathrm{PO}_{4}, 2.5 \mathrm{~mm} \mathrm{KCl}, 0.5$ $\mathrm{mm} \mathrm{CaCl} 2,7.0 \mathrm{~mm} \mathrm{MgCl} 2,25.0 \mathrm{~mm}$ glucose, $11.6 \mathrm{~mm}$ ascorbic acid, and $3.1 \mathrm{~mm}$ pyruvic acid, gassed with $95 \% \mathrm{O}_{2}$ and $5 \% \mathrm{CO}_{2}$ ). $300 \mu \mathrm{m}$ coronal slices containing the GPe were cut in dissection buffer using a HM650 Vibrating-blade Microtome (Thermo Fisher Scientific). Slices were immediately transferred to a storage chamber containing ACSF at $34^{\circ} \mathrm{C}$. After 40-min recovery time, slices were transferred to room temperature (RT; $20-24^{\circ} \mathrm{C}$ ) and perfused with gassed ACSF constantly throughout recording.

Whole-cell patch clamp recording was performed as previously described (Li et al., 2013). Briefly, recording from GPe neurons was obtained with Multiclamp 700B amplifiers and pCLAMP 10 software (Molecular Devices), and was visually guided using an Olympus BX51 microscope equipped with both transmitted and epifluorescence light sources (Olympus Corporation). The external solution was ACSF. The internal solution contained $115 \mathrm{~mm}$ cesium methanesulfonate, $20 \mathrm{~mm}$ CsCl, 10 mм HEPES, 2.5 mм $\mathrm{MgCl}_{2}$, 4 mм Na $\mathrm{N}_{2} \mathrm{ATP}, 0.4 \mathrm{~mm} \mathrm{Na}_{3} \mathrm{GTP}, 10$ mM sodium phosphocreatine, and 0.6 mM EGTA (pH 7.2).

As the acute slices were prepared from Sst-IRES-Cre mice in which $\mathrm{Sst}^{+} \mathrm{CeA}$ neurons were infected with AAV expressing ChR2-YFP, to evoke synaptic transmission onto GPe neurons driven by the $\mathrm{Sst}^{+}$neurons, a blue light was used to stimulate ChR2-expressing axons originating from the $\mathrm{Sst}^{+}$neurons. The light source was a single-wavelength LED system $(\lambda=470 \mathrm{~nm}$; CoolLED) connected to the epifluorescence port of the Olympus BX51 microscope. A light pulse of $1 \mathrm{~ms}$, triggered by a TTL signal from the Clampex software, was delivered every $10 \mathrm{~s}$ to evoke synaptic responses. Evoked IPSCs were recorded at a holding potential of $0 \mathrm{mV}$ and in ACSF with $100 \mu \mathrm{M}$ AP5 and $10 \mu \mathrm{M}$ CNQX added to block excitatory synaptic transmission. Synaptic responses were low-pass filtered at $1 \mathrm{kHz}$ and were analyzed using pCLAMP 10 software. Evoked IPSCs were quantified as the mean current amplitude from 50 to $60 \mathrm{~ms}$ after stimulation.

\section{Immunohistochemistry}

For histology analysis, mice were anesthetized with Euthasol $(0.2 \mathrm{ml}$; Virbac) and perfused transcardially with $30-\mathrm{ml}$ cold PBS followed by 30 $\mathrm{ml} 4 \%$ paraformaldehyde (PFA) in PBS. Brains were removed immediately from the skull and placed in PFA for at least $24 \mathrm{~h}$ and then in $30 \%$ sucrose in PBS solution for $24 \mathrm{~h}$ for cryoprotection. Coronal sections $(50 \mu \mathrm{m})$ were cut using a freezing microtome (Leica SM 2010R, Leica) and placed in PBS in 12-well plates. Brain sections were first washed in PBS $(3 \times 5 \mathrm{~min})$, incubated in PBST $(0.3 \%$ Triton X-100 in PBS) for $30 \mathrm{~min}$ at RT and then washed with PBS $(3 \times 5 \mathrm{~min})$. Next, sections were blocked in 5\% normal goat serum in PBST for $30 \mathrm{~min}$ at RT and then incubated with the primary antibody for $12 \mathrm{~h}$ at $4^{\circ} \mathrm{C}$. Sections were washed with PBS $(5 \times 15 \mathrm{~min})$ and incubated with the fluorescent secondary antibody at RT for $2 \mathrm{~h}$. After washing with PBS $(5 \times 15 \mathrm{~min})$, sections were mounted onto slides with Fluoromount-G (eBioscience). Images were taken using an LSM 710 laser-scanning confocal microscope (Carl Zeiss).

The primary antibodies used in this study were: chicken anti-GFP (Aves Labs, catalog \#GFP1020, lot \#GFP697986), rabbit anti-RFP (Rockland, catalog \#600-401-379, lot \#34135). The fluorophore-conjugated secondary antibodies used were Alexa Fluor 488 donkey antichicken IgG $(\mathrm{H}+\mathrm{L})$, Alexa Fluor 488 goat anti-rabbit $\operatorname{IgG}(\mathrm{H}+\mathrm{L})$, and Alexa Fluor 555 goat anti-rabbit IgG (H+L; Life Technologies).

\section{Fluorescent in situ hybridization (FISH)}

Single molecule FISH (smFISH; ACDBio, RNAscope) was used to detect the expression of Sst and Prkcd mRNAs in the CeA of adult mice, which were injected in the GPe with CTB-555. Five days after CTB injection, mice were first anesthetized under isoflurane and then decapitated. Their brain tissue was first embedded in cryomolds (Sakura Finetek, Ref 4566) filled with M-1 Embedding Matrix (Thermo Scientific, catalog \#1310) then quickly fresh-frozen on dry ice. The tissue was stored at $-80^{\circ} \mathrm{C}$ until it was sectioned with a cryostat. Cryostat-cut sections (16$\mu \mathrm{m})$ containing the CeA were collected and quickly stored at $-80^{\circ} \mathrm{C}$ until processed. Hybridization was conducted using the RNAscope kit (ACDBio).

The day of the experiment, frozen sections were postfixed in 4\% PFA in RNA-free PBS (hereafter referred to as PBS) at RT for $15 \mathrm{~min}$, then washed in PBS, dehydrated using increasing concentrations of ethanol in water (50\%, once; $70 \%$, once; $100 \%$, twice; $5 \mathrm{~min}$ each). Sections were then dried at RT and incubated with Protease IV for $30 \mathrm{~min}$ at RT. Sections were washed in PBS three times ( 5 min each) at RT, then hybridized. Probes against Sst (catalog \#404631, dilution 1:50) and Prkcd (catalog \#441791, dilution 1:50) were applied to CeA sections. Hybridization was conducted for $2 \mathrm{~h}$ at $40^{\circ} \mathrm{C}$. After that, sections were washed twice in PBS ( 2 min each) at RT, then incubated with three consecutive rounds of amplification reagents $\left(30,15\right.$, and $30 \mathrm{~min}$, at $\left.40^{\circ} \mathrm{C}\right)$. After each amplification step, sections were washed twice in PBS ( $2 \mathrm{~min}$ each) at RT. Finally, fluorescence detection was conducted for $15 \mathrm{~min}$ at $40^{\circ} \mathrm{C}$. The red channel was left free for detection of CTB-555 fluorescence. Sections were then washed twice in PBS, incubated with DAPI for $2 \mathrm{~min}$, washed twice in PBS ( 2 min each), then mounted with coverslip using mounting medium. Images were acquired using an LSM780 confocal microscope equipped with $20 \times, 40 \times$, or $63 \times$ lenses, and visualized and processed using ImageJ and Adobe Illustrator.

\section{Behavioral tasks}

\section{Auditory fear conditioning}

We followed standard procedures for conventional auditory fear conditioning (Li et al., 2013; Penzo et al., 2014, 2015; Yu et al., 2017). Briefly, mice were initially handled and habituated to a conditioning cage, which was a Mouse Test Cage $(18 \times 18 \times 30 \mathrm{~cm})$ with an electrifiable floor connected to a H13-15 shock generator (Coulbourn Instruments). The Test Cage was placed inside a sound attenuated cabinet (H10-24A; Coulbourn Instruments). Before each habituation and conditioning session, the Test Cage was wiped with $70 \%$ ethanol. The cabinet was illuminated with white light during habituation and conditioning sessions.

During habituation, two $4-\mathrm{kHz} 75-\mathrm{dB}$ tones and two $12-\mathrm{kHz} 75-\mathrm{dB}$ tones, each of which was $30 \mathrm{~s}$ in duration, were delivered at variable intervals within an 8-min session. During conditioning, mice received three presentations of the $4-\mathrm{kHz}$ tone [conditioned stimulus $\left(\mathrm{CS}^{+}\right)$], each of which co-terminated with a 2 -s $0.7-\mathrm{mA}$ foot shock (unless otherwise stated), and three presentations of the $12-\mathrm{kHz}$ tone, which were not paired with foot shocks $\left(\mathrm{CS}^{-}\right)$. The $\mathrm{CS}^{+}$and $\mathrm{CS}^{-}$were interleaved pseudo-randomly, with variable intervals between 30 and $90 \mathrm{~s}$ within a 10 -min session. The test for fear memory (retrieval) was performed $24 \mathrm{~h}$ following conditioning in a novel context, where mice were exposed to two presentations of $\mathrm{CS}^{+}$and $\mathrm{CS}^{-}(>120$-s inter-CS interval). The novel context was a cage with a different shape $(22 \times 22 \times 21 \mathrm{~cm})$ and floor texture compared with the conditioning cage, and was illuminated with infrared light. Before each use the floor and walls of the cage were wiped clean with $0.5 \%$ acetic acid to make the scent distinct from that of the conditioning cage.

For optogenetic manipulation with stGtACR1 during fear conditioning, blue light ( $473 \mathrm{~nm}, 5 \mathrm{~mW}$; 4-s square pulse) was delivered via tethered patchcord to the implanted optical fibers. The onset of the light coincided with the onset of US (2-s $0.7-\mathrm{mA}$ foot shock) presentation. For optogenetic manipulation with ChR2 during fear conditioning, blue light ( $473 \mathrm{~nm}, 5 \mathrm{~mW}$; $30-\mathrm{Hz}, 5-\mathrm{ms}$ pulses for $2 \mathrm{~s}$ ) was delivered via tethered patchcord to the implanted optical fibers, coinciding with the presentation of US (2-s 0.4-mA foot shock).

Animal behavior was videotaped with a monochrome CCD-camera (Panasonic WV-BP334) at $3.7 \mathrm{~Hz}$ and stored on a personal computer. 
The FreezeFrame software (Coulbourn Instruments) was used to control the delivery of both tones and foot shocks. Freezing behavior was analyzed with FreezeFrame software (Coulbourn Instruments) for the TeLC experiment. For subsequent fiber photometry and optogenetic experiments, Ethovision XT 5.1 (Noldus Information Technologies) was used to track the animal, and freezing was calculated using a custom Matlab script for improved tracking while avoiding the influence by patchcords and optic fibers attached to animal's head. Baseline freezing levels were calculated as the average freezing during the first $100 \mathrm{~s}$ of the session before any stimuli were presented, and freezing to the auditory stimuli was calculated as the average freezing during the tone presentation. The average of the freezing responses to two $\mathrm{CS}^{+}$or $\mathrm{CS}^{-}$presentations during recall was used as an index of fear. Discrimination Index was calculated as the difference between freezing to the $\mathrm{CS}^{+}$and $\mathrm{CS}^{-}$, normalized by the sum of freezing to both tones.

Real-time place preference or aversion test

Freely moving mice were habituated to a two-sided chamber (made from Plexiglas; $23 \times 33 \times 25 \mathrm{~cm}$ for each side) for $10 \mathrm{~min}$, during which baseline preference to each side was assessed. During the first test session ( $10 \mathrm{~min}$ ), one side of the chamber was designated the photo-stimulation side, and mice were placed in the middle to start the experiment. Once the mouse entered the stimulation side, photo-stimulation (5-ms pulses, $30 \mathrm{~Hz}, 10 \mathrm{~mW}$; measured at the tip of optic fibers) with a 473-nm laser (OEM Laser Systems Inc.) was turned on, and was turned off on the mouse exiting the stimulation side. In the second test session (10 min) this procedure was repeated, with the opposite side being the stimulation side. Animal behavior was videotaped with a CCD camera (C930, Logitech) and tracked with Ethovision, which was also used to control the laser stimulation and extract behavioral parameters (position, time, distance, and velocity).

\section{In vivo fiber photometry and data analysis}

A commercial fiber photometry system (Neurophotometrics Ltd.) was used to record GCaMP6f signals in GPe-projecting CeA neurons in vivo in behaving animals through an optical fiber $(200-\mu \mathrm{m}$ fiber core diameter, 5.0-mm length, $0.37 \mathrm{NA}$; Inper) implanted in the CeA. A patch cord (fiber core diameter, $200 \mu \mathrm{m}$; Doric Lenses) was used to connect the photometry system with the implanted optical fiber. The intensity of the blue light $(\lambda=470 \mathrm{~nm})$ for excitation was adjusted to $\sim 20 \mu \mathrm{W}$ at the tip of the patch cord. Emitted GCaMP6f fluorescence was bandpass filtered and focused on the sensor of a CCD camera. Photometry signals and behavioral events were aligned based on an analog TTL signal generated by a Bpod. Mean values of signals from a region of interest were calculated and saved using Bonsai software (Bonsai), and exported to MATLAB for further analysis.

To correct for slow baseline drifting caused by photobleaching, a time-dependent baseline $\mathrm{F}_{0}(\mathrm{t}$ ) was computed as described previously (Jia et al., 2011). The percentage $\Delta \mathrm{F} / \mathrm{F}$ was calculated as $100 \times\left(\mathrm{F}(\mathrm{t})-\mathrm{F}_{0}(t)\right) /$ $\mathrm{F}_{0}(t)$, where $\mathrm{F}(\mathrm{t})$ is the raw fluorescence signal at time $t$. After baseline drift correction, the fluorescence signals were $z$-scored relative to the mean and standard deviation of the signals in the entire trial. In this experiment, we simultaneously recorded both the calcium-dependent signals and the isosbestic signals from the GCaMP6, with the latter serving to monitor potential motion artifacts as previously described (Kim et al., 2016). Average calcium responses for each animal were calculated using the $z$-scored $\Delta \mathrm{F}$ signal during the 2-s shock period averaged across all three trials. To calculate the trial-by-trial correlations between GCaMP6 signals and movement velocity, the $z$-scores for GCaMP6 signals or velocity in each trial were calculated based on the mean and standard deviation of that trial. All trials from each mouse we pooled for the analysis.

\section{Experimental design and statistical analysis}

All statistics are indicated where used. Statistical analyses were performed with GraphPad Prism Software (GraphPad Software). Normality was tested by D'Agostino-Pearson or Shapiro-Wilk normality tests. All behavioral experiments were controlled by computer systems, and data were collected and analyzed in an automated and unbiased way. Virus- injected animals in which the injection site was incorrect were excluded. No other mice or data points were excluded.

\section{Results}

A subpopulation of $\mathrm{CeA}$ neurons send projections to the GPe It has been reported that the CeA sends projections to the GPe (Shinonaga et al., 1992). We started to verify this result by using a retrograde tracing approach (Fig. $1 A$ ). We injected a retrograde adeno-associated virus (AAVrg) encoding the Cre recombinase (AAVrg-Cre; Keyes et al., 2020) into the GPe of LSL-H2B-GFP reporter mice (He et al., 2012), which express the fluorescent protein $\mathrm{H} 2 \mathrm{~B}-\mathrm{GFP}$ (nuclear GFP) in a Cre-dependent manner. This approach led to the labeling of many neurons in the CeA (Fig. $1 B$ ), confirming the existence of the CeA-GPe pathway.

To determine the main composition of $\mathrm{CeA}$ neurons projecting to the GPe, we injected the GPe in wild-type mice with the retrograde tracer CTB conjugated with Alexa Fluor 555 (CTB555; Fig. 1C). We subsequently assessed the expression of Sst and Prkcd (which encodes PKC- $\delta$ ) in the CTB-labeled GPe-projecting CeA neurons using smFISH (Fig. 1D). This approach revealed that the vast majority of $\mathrm{GPe}$-projecting $\mathrm{CeA}$ neurons expresses Sst ( $93 \pm 3 \%$; mean \pm SEM), whereas only small portions of these neurons express Prkcd (6士1\%), both Sst and

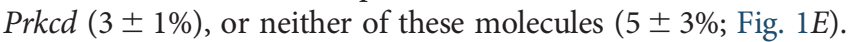
Similarly, retrograde tracing with CTB in Sst-IRES-Cre;Ai14 mice, in which Sst ${ }^{+}$cells are labeled with the fluorescent protein tdTomato (Madisen et al., 2010), showed that almost all the GPe-projecting CeA neurons are $\mathrm{Sst}^{+}(92 \pm 2 \%$; $n=4$ mice; Fig. $1 F, G)$.

In a complimentary experiment, we visualized the CeA-GPe pathway using an anterograde tracing approach. An AAV expressing the fluorescent protein mCherry in a Cre-dependent manner was injected into the CeA of Sst-IRES-Cre mice to label $\mathrm{Sst}^{+}$CeA neurons (Fig. $1 \mathrm{H}$ ). Four to five weeks later, we examined the brain sections from these mice for axon fibers originating from the infected $\mathrm{Sst}^{+} \mathrm{CeA}$ neurons. Dense fibers were identified in the dorsal part of the GPe (Fig. 1I). Together, these results demonstrate that projections from the $\mathrm{CeA}$ to the GPe originate predominantly from $\mathrm{Sst}^{+}$neurons.

Next, we examined the functional connectivity between the $\mathrm{CeA}$ and the GPe (Fig. 1J-L). We introduced the light-gated cation channel channelrhodopsin (ChR2) selectively into $\mathrm{Sst}^{+} \mathrm{CeA}$ neurons of Sst-IRES-Cre mice, and used these mice to prepare acute brain slices containing the GPe, in which we recorded synaptic responses in neurons in response to light-simulation of the axons originating from $\mathrm{Sst}^{+} \mathrm{CeA}$ neurons (Fig. 1J,K). About half of the neurons (five out of 12) recorded in the GPe showed fast light-evoked inhibitory synaptic responses (Fig. $1 \mathrm{~L}$ ), indicating that $\mathrm{Sst}^{+} \mathrm{CeA}$ neurons provide monosynaptic inhibition onto a subset of GPe neurons.

It is known that $\mathrm{Sst}^{+} \mathrm{CeA}$ neurons send projections to many downstream structures (Penzo et al., 2014; Fadok et al., 2017; Yu et al., 2017; Ahrens et al., 2018; Zhou et al., 2018; Li, 2019; Ye and Veinante, 2019). Therefore, we examined whether the GPeprojecting $\mathrm{Sst}^{+}$neurons send collateral projections to a major target of the CeA, the bed nucleus of the stria terminalis (BNST), because our recent study shows that BNST-projecting CeA neurons are also predominantly $\mathrm{Sst}^{+}$, and these neurons play a critical role in anxiety-related behaviors (Ahrens et al., 2018). To this end, we injected both the GPe and the BNST in the same mice with CTB conjugated with different fluorophores, such that GPeprojecting neurons and BNST-projecting neurons in the CeA 
A

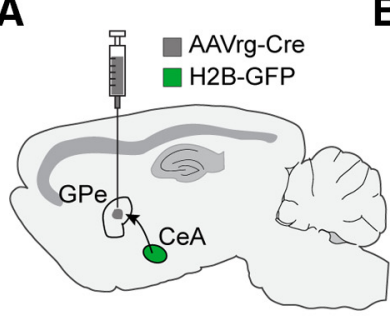

LSL-H2B-GFP mouse
B

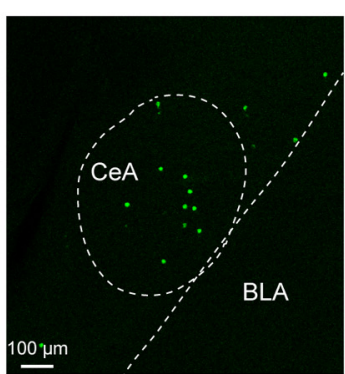

C

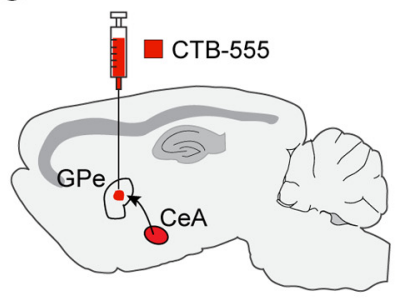

D

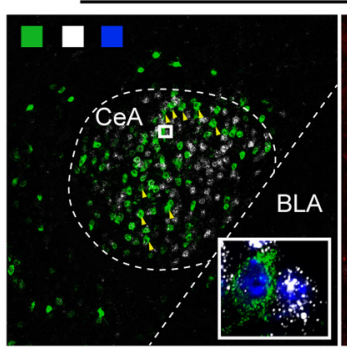

$\mathbf{F}$

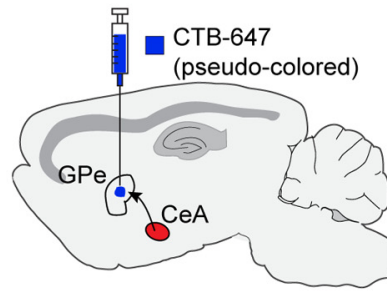

Sst-Cre:Ai14 mouse

H

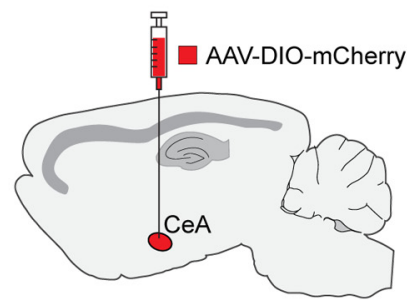

Sst-Cre mouse

J

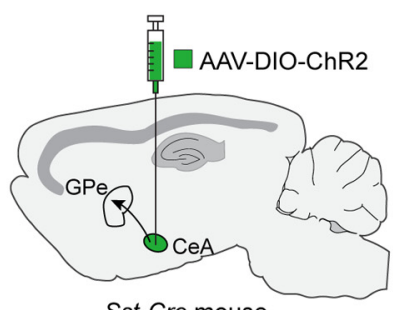

Sst-Cre mouse $\square$ sst
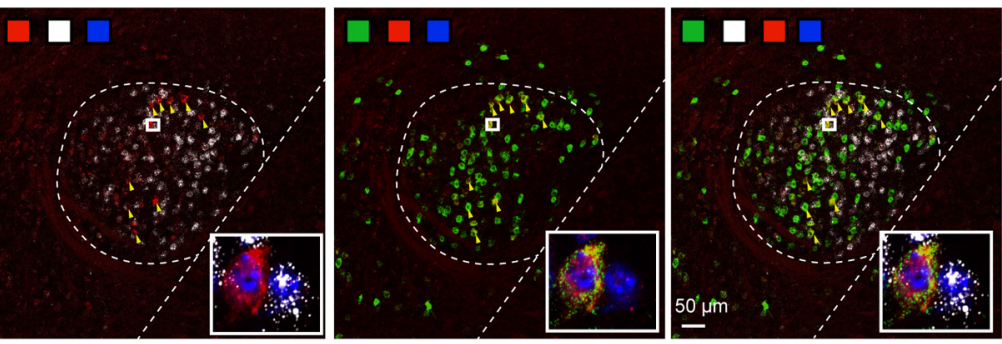

G
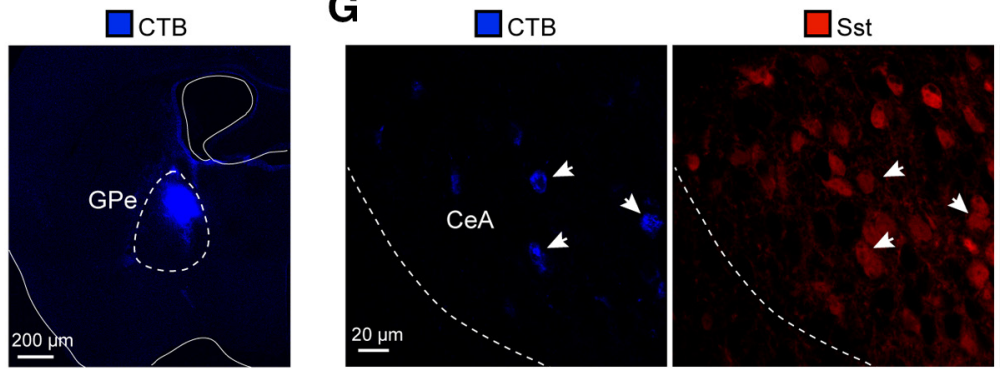

I
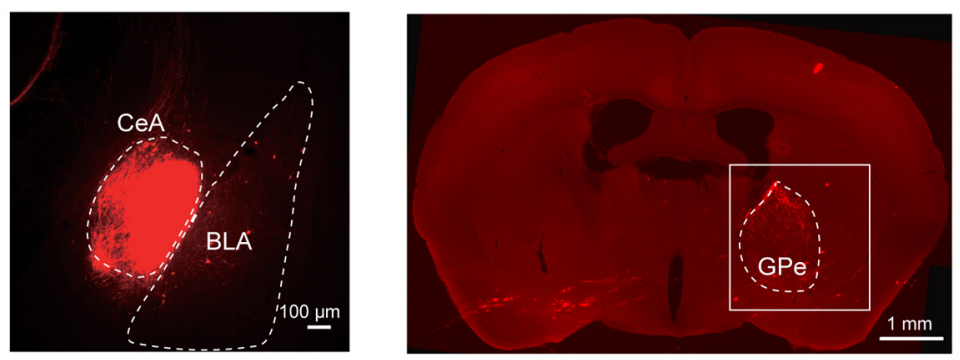

K

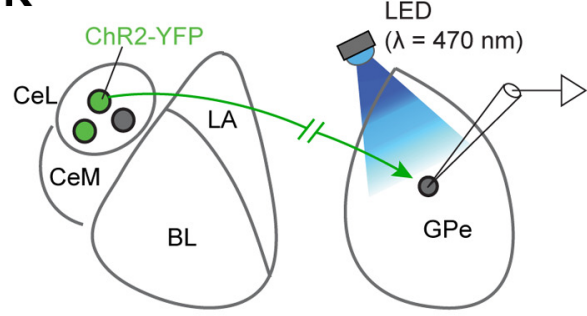

Sst-Cre mouse
L

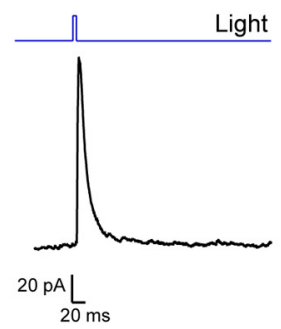

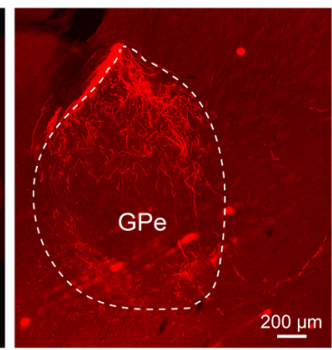

$\square$ СTB $\square$ DAPI

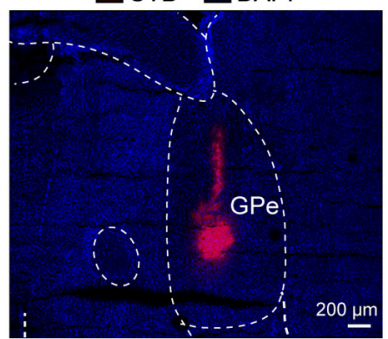

E

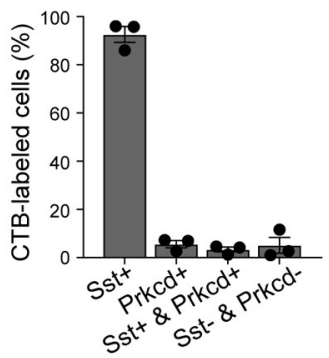

$\square$ Sst / $\square$ CTB

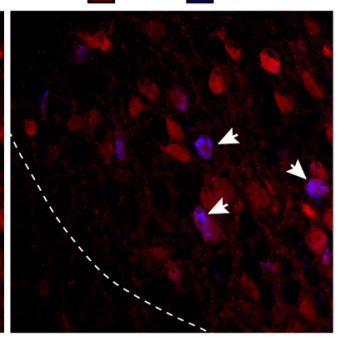

$\underline{200 \mu m}$

Figure 1. Characterization of CeA-to-GPe projections. $\boldsymbol{A}, \boldsymbol{B}$, Schematic of the approach $(\boldsymbol{A})$ and a representative image showing the retrogradely-labeled $\mathrm{H} 2 \mathrm{~B}^{+}$cells in the CeA $(\boldsymbol{B} ; n=2$ mice). $\boldsymbol{C}$, Schematic of the approach (left) and a representative image showing the target area of CTB injection in the GPe (right). $\boldsymbol{D}$, Confocal images of a coronal brain section containing the CeA from a representative mouse in which CTB was injected into the GPe $(\mathbf{C})$, showing the distribution of GPe-projecting CeA neurons labeled with (TB, and the distribution of Sst and Prkcd expression detected with smFISH. Insets, High-magnification images of the boxed areas in each of the images. $\boldsymbol{E}$, Quantification of the percentage distribution of different types of CeA neurons that project to the GPe ( $n=3$ mice). $\boldsymbol{F}$, Schematic of the approach (left) and a representative image showing the target area of CTB injection in the GPe (right). $\mathbf{G}$, Confocal images of a coronal brain section containing the CeA from a representative Sst-Cre;Ai14 mouse in which CTB was injected into the GPe (F), showing the distribution of GPe-projecting CeA neurons labeled with $\mathrm{CTB}$, and the distribution of $\mathrm{Sst}^{+}$neurons labeled with tdTomato. $\boldsymbol{H}$, Schematic of the approach (left) and a representative image showing the viral infection of Sst ${ }^{+}$CeA neurons (red; right). $\boldsymbol{I}$, left, Image of a coronal brain section containing the GPe from a representative Sst-Cre mouse in which Sst ${ }^{+}$CeA neurons were labeled with mCherry $(\boldsymbol{H})$. Right, Higher-magnification image 
were labeled with distinct colors (Fig. 2A$C)$. Notably, we found almost no doubly labeled neurons in the CeA in these mice $(<1 \%$; Fig. $2 D)$, indicating that GPe-projecting and BNST-projecting CeA neurons are distinct populations.

\section{GPe-projecting CeA neurons are necessary for fear learning}

As CeA neurons, particularly the $\mathrm{Sst}^{+}$neurons (Fadok et al., 2018; Li, 2019), and the GPe (Blanchard et al., 1981; Murphy et al., 2003; Talalaenko et al., 2006; Kertes et al., 2009; Sztainberg et al., 2011; Hattingh et al., 2012; Ipser et al., 2013) have been implicated in processing negative affects including fear, we set out to examine the role of GPe-projecting $\mathrm{CeA}$ neurons in Pavlovian fear conditioning. To determine whether GPe-projecting CeA neurons are necessary for fear conditioning, we selectively blocked neurotransmitter release from these neurons with the tetanus toxin light chain (TeLC; Murray et al., 2011). To this end, we used an intersectional viral strategy in wild-type mice, in which we bilaterally injected the GPe with the AAVrg-Cre and the CeA with an AAV expressing TeLC-GFP, or GFP (as the control), in a Cre-dependent manner (Fig. $3 A, B$ ). Four weeks after viral injection, both the TeLC group and the GFP control group were trained in an auditory fear conditioning paradigm whereby one sound (the $\mathrm{CS}^{+}$) was paired with a foot shock (the US), and another sound (the neutral sound, or $\mathrm{CS}^{-}$) was not paired with any outcome (Fig. $3 C$; Materials and Methods).

Remarkably, blocking transmitter release from GPe-projecting CeA neurons with TeLC completely abolished the conditioned freezing induced by $\mathrm{CS}^{+}$during a memory retrieval test $24 \mathrm{~h}$ after the conditioning (conditioning: $F_{(1,15)}=4.47, p=0.052$; retrieval, $\mathrm{CS}^{+}$trials: $F_{(1,15)}=25.21,{ }^{\star * *} p=0.0002 ;{ }^{\star * \star} p<0.001$, ${ }^{* * * *} p<0.0001$; retrieval, $\mathrm{CS}^{-}$trials: $F_{(1,15)}=14.41, p=0.060$; twoway ANOVA with repeated measures, followed by Sidak's test; Fig. 3C). Furthermore, this manipulation also reduced the responses of the mice to foot shocks, as indicated by a reduction in the peak velocity of shock-induced movements (peak velocity: $F_{(1,75)}=6.359,{ }^{\star} p=0.014$; distance moved: $F_{(1,75)}=1.619, p=$ 0.210; two-way ANOVA; Fig. $3 D$ ). These results indicate that GPe-projecting CeA neurons are indispensable for fear conditioning, and suggest that these neurons have a role in processing information about the US.

of the boxed area in the left, showing the distribution of axon fibers in the GPe that originate from Sst ${ }^{+}$CeA neurons. This experiment was repeated in three mice. J, Schematic of the approach. $\boldsymbol{K}$, Schematic of the in vitro recording configuration in acute slices. $\boldsymbol{L}$, left, Average trace of synaptic currents recorded from a representative neuron in the GPe. Synaptic currents were evoked by optogenetic activation of axon fibers originating from $\mathrm{Sst}^{+}$CeA neurons, and were recorded at a holding potential of $0 \mathrm{mV}$ in the presence of $100 \mu \mathrm{m} \mathrm{AP5}$ and 10 $\mu \mathrm{m}$ CNQX to isolate IPSCS (see Materials and Methods). The upward square pulse in the blue trace on top of the synaptic current indicates the timing of photo-stimulation. Right, Quantification of the amplitude of the evoked IPSCs (five out of 12 cells recorded from five mice had evoked IPS(S). Data in $\boldsymbol{E}, \boldsymbol{L}$ are presented as mean \pm SEM.

\section{GPe-projecting CeA neurons convey information about the US during fear conditioning}

To further understand the in vivo function of GPe-projecting $\mathrm{CeA}$ neurons, we recorded the activities of these neurons in behaving mice (Fig. 4A-I). For this purpose, we introduced the genetically encoded calcium indicator GCaMP6 (Chen et al., 2013) into these neurons using the above described intersectional viral strategy, in which we injected the AAVrg-Cre unilaterally into the GPe (Figs. $1 C, D, 3 A$ ), and an AAV expressing GCaMP6 in a Cre-dependent manner into the ipsilateral CeA (Fig. 4A,B) in wild-type mice. These mice were then implanted with optical fibers above the infected area in the CeA (Fig. 4A,B,I). Four weeks after the surgery, we trained the mice in auditory fear conditioning as described above (Fig. 3C), and verified that these mice showed discriminative learning as indicated by higher freezing levels to $\mathrm{CS}^{+}$than to $\mathrm{CS}^{-}$during the memory retrieval test $\left(F_{(1.314,6.570)}=15.37, p=0.005,{ }^{*} p=0.023,{ }^{* *} p=0.005\right.$; oneway ANOVA followed by Tukey's test; Fig. $4 C$ ).

We recorded bulk GCaMP6 signals from the infected GPeprojecting CeA neurons in these animals with fiber photometry (Yu et al., 2016) throughout fear conditioning (Fig. 4A-E). In this experiment, we simultaneously recorded both the calciumdependent signals and the isosbestic signals from the GCaMP6 (Fig. 4D), with the latter serving to monitor potential motion artifacts (Kim et al., 2016). Notably, we found that GPe-projecting $\mathrm{CeA}$ neurons showed potent excitatory response to US (shock) presentations during conditioning, but little response to $\mathrm{CS}^{+}$(or $\mathrm{CS}^{-}$) presentations during either conditioning or the memory retrieval test [Fig. $4 D, E$; conditioning (left), $F_{(3,15)}=$ 80.30, $p<0.0001,{ }^{* * *} p<0.0001$; retrieval (right), n.s. (nonsignificant), $p=0.57$; two-way ANOVA followed by Tukey's test; Fig. $4 E)$. This result is in sharp contrast with those from $\mathrm{Sst}^{+} \mathrm{CeA}$ neurons with unknown projection targets, which show robust excitatory responses to CS after fear conditioning as assessed by in vivo single unit recording (Fadok et al., 2017) or fiber photometry (Yu et al., 2016). There was no significant correlation 
A

AAVrg-Cre

AAV-DIO-TeLC-GFP

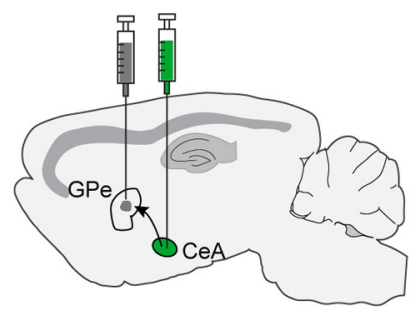

C

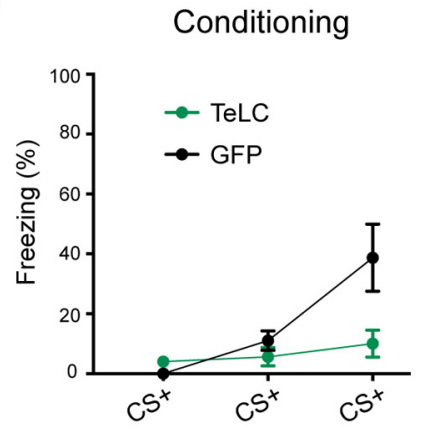

B
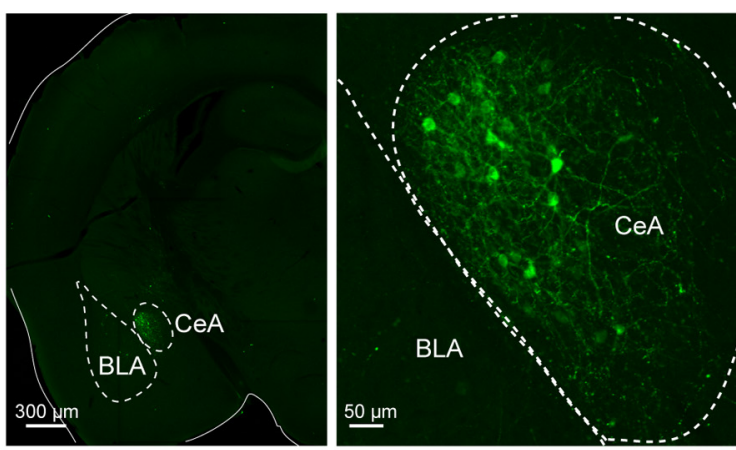

Retrieval

\section{D}
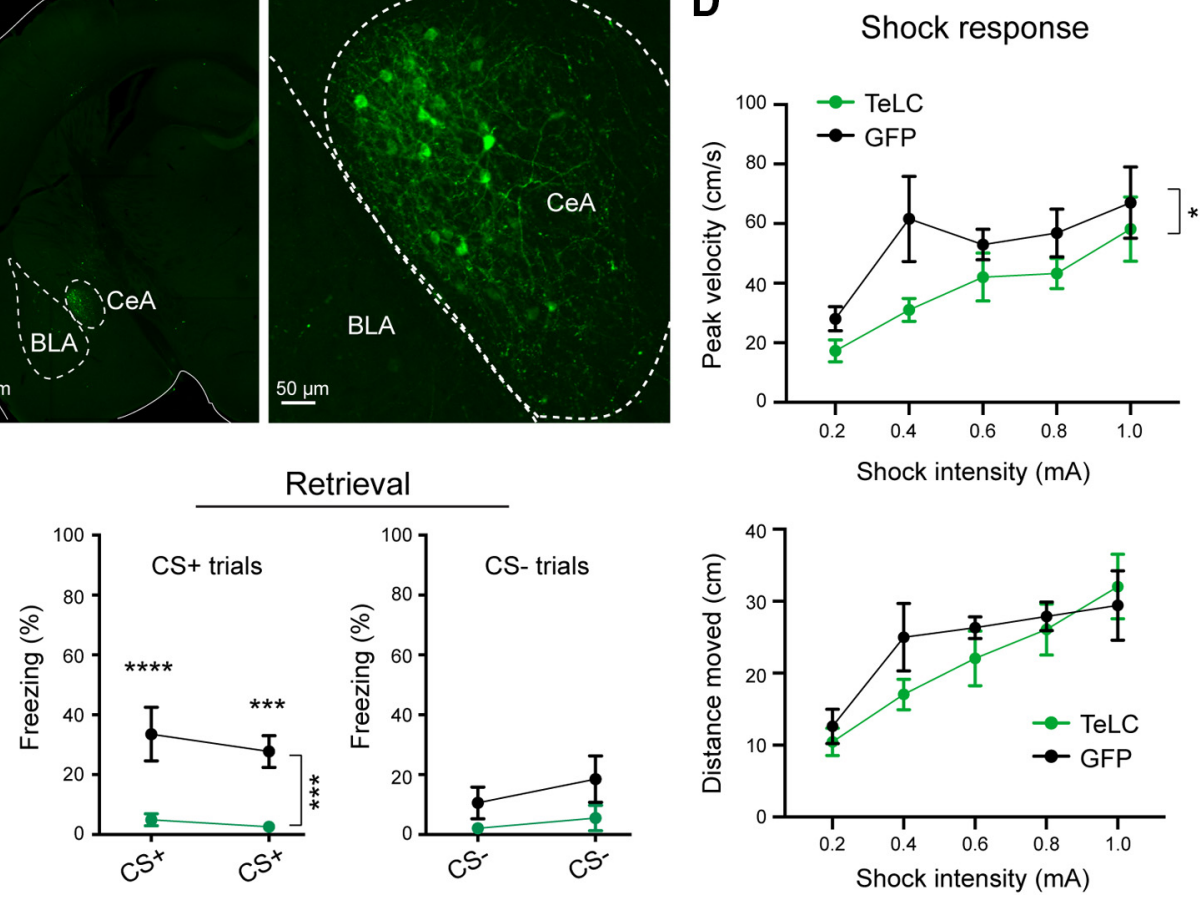

Figure 3. Inhibition of GPe-projecting CeA neurons blocks fear conditioning. $\boldsymbol{A}$, Schematic of the approach. $\boldsymbol{B}$, Representative confocal images showing the GPe-projecting CeA neurons expressing TeLC. On the right is a higher-magnification image of the amygdala area on the left. C, Freezing behavior in mice in which GPe-projecting CeA neurons expressed TeLC ( $n=11)$ or GFP $(n=6)$, during conditioning (left) and retrieval (right) sessions. $\boldsymbol{D}$, Peak velocity (top) and distance moved (bottom) for movements in mice in $\boldsymbol{C}$, in response to shocks of varying intensities. Data in $\boldsymbol{C}, \boldsymbol{D}$ are presented as mean \pm SEM. ${ }^{*} p<0.05,{ }^{* * *} p<0.001,{ }^{* * * *} p<0.0001$.

between GCaMP6 signals and movement velocity during either the shock ( $r=-0.3483, p=0.1566$, Spearman correlation) or baseline ( $r=-0.2997, p=0.2269$, Spearman correlation) period across animals (Fig. $4 F$ ), suggesting that the activity of GPe-projecting $\mathrm{CeA}$ neurons does not represent movement vigor. Further examination revealed that the responses of GPe-projecting CeA neurons were significantly higher to stronger shocks than to weaker ones $\left({ }^{\star} p=0.031\right.$, Wilcoxon paired $t$ test; Fig. $4 G$, $H$ ), indicating that the responses represent shock intensity. These results point to the possibility that GPe-projecting CeA neurons play an important role in processing US information thereby instructing learning in fear conditioning.

\section{GPe-projecting CeA neuron activity during US presentation is required for learning}

To determine whether the excitatory response of GPe-projecting CeA neurons evoked by US during fear conditioning is required for learning, we sought to transiently inhibit these neurons only during the presentation of the US (Fig. 5A-F). To achieve this goal, we introduced the light sensitive Guillardia theta anionconducting channelrhodopsin 1 (GtACR1; Govorunova et al., 2015; Mahn et al., 2018) selectively into GPe-projecting CeA neurons using the intersectional viral strategy described above (Figs. $1 A, B, 3 A, B, 4 A, B$ ). Specifically, we injected the AAVrg-Cre bilaterally into the GPe and an AAV expressing GtACR1, or GFP, in a Cre-dependent manner bilaterally into the CeA, followed by implanting optical fibers above the infected areas (Fig. $5 A, F)$.

Four weeks following viral injection, both the GtACR1 group and the GFP group (which served as the control) were trained in the auditory fear conditioning paradigm (Fig. 5B). During conditioning, square pulses of blue light, covering the duration of each of the three US presentations, were delivered to the CeA through the implanted optical fibers (Fig. 5B). Notably, we found that this manipulation caused a decrease in $\mathrm{CS}^{+}$-induced conditioned freezing behavior in the GtACR1 mice compared with the GFP mice in the retrieval test $24 \mathrm{~h}$ after fear conditioning (conditioning: $F_{(1,12)}=0.117, p>0.05$; retrieval, $\mathrm{CS}^{+}$trials: $F_{(1,12)}=15.65$, ${ }^{\star *} p=0.002$; ${ }^{*} p<0.05,{ }^{* *} p<0.010$; retrieval, $\mathrm{CS}^{-}$trials: $F_{(1,12)}=$ $0.010, p>0.05$; two-way ANOVA with repeated measures, followed by Sidak's test; Fig. 5B). As a result, the ability to discriminate between $\mathrm{CS}^{+}$and $\mathrm{CS}^{-}$, quantified as the discrimination index, was also reduced in the GtACR1 mice $\left(t_{(10.51)}=2.329\right.$, ${ }^{\star} p=0.041$, Welch's $t$ test; Fig. $5 C$ ). We next tested these mice in a real-time place preference or aversion (RTPP or RTPA, respectively) task, in which the photoinhibition was contingent on entering one side of a chamber containing two compartments (Fig. 5D). The two groups of animals behaved similarly in this task, showing no preference or aversion to either side of the chamber $\left(F_{(1,12)}=2.135, p>0.05\right.$; two-way ANOVA with repeated measures; Fig. $5 E$ ). This observation suggests that photoinhibition of $\mathrm{GPe}$-projecting $\mathrm{CeA}$ neurons is not inherently aversive or rewarding. These results indicate that the activities of GPe-projecting CeA neurons during US presentation are required for memory formation in fear conditioning.

\section{Activation of GPe-projecting CeA neurons during US presentation promotes fear learning}

Given that inhibition of GPe-projecting CeA neurons specifically during US presentation impaired learning (Fig. 5), it follows that the opposite manipulation, i.e., activation of these neurons specifically during US presentation, might enhance learning in fear conditioning. To test this idea, we introduced ChR2, or GFP, bilaterally into $\mathrm{GPe}$-projecting $\mathrm{CeA}$ neurons of wild-type mice using the intersectional viral strategy, followed by optical fiber implantation in the CeA as described above (Figs. $3 A, B, 4 A, B$, 
A

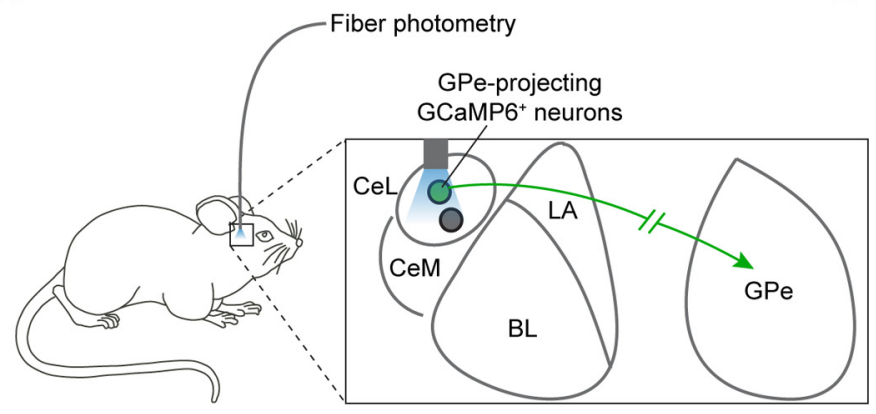

B

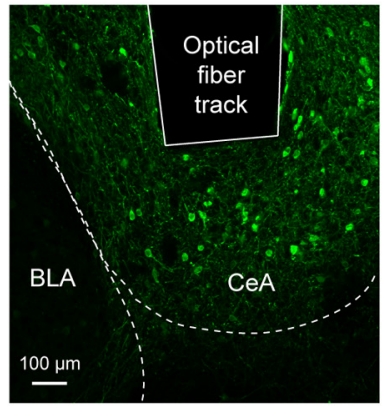

C

Memory retrieval

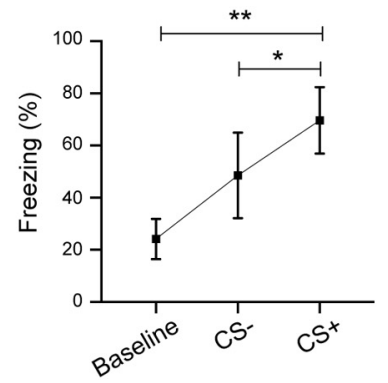

E

$\mathrm{CS}+$ trials
Retrieval

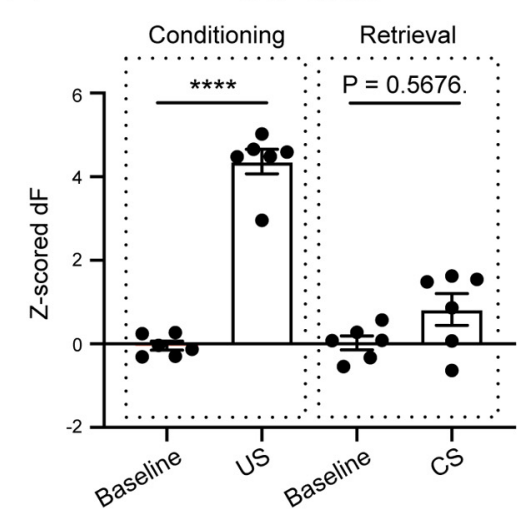

F
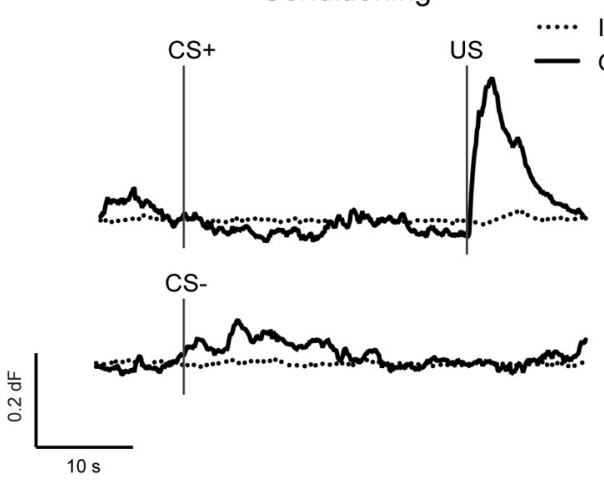

sosbestic signal
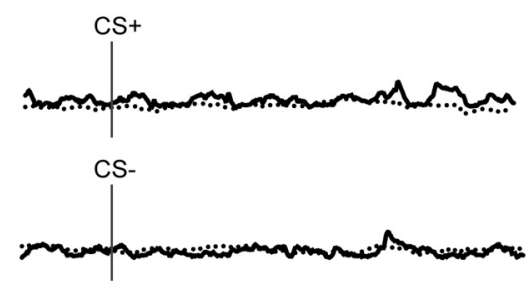

G

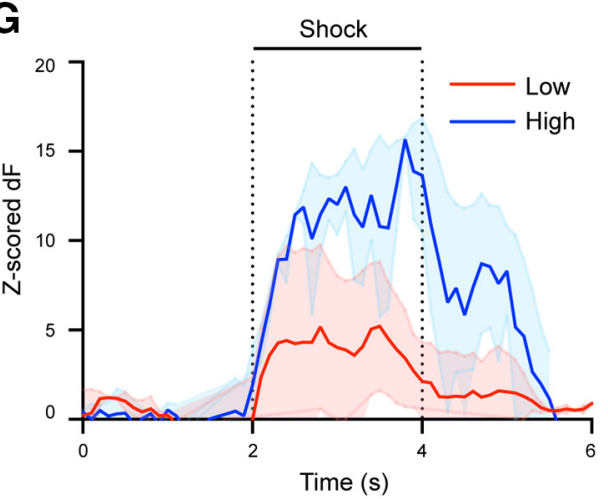

H

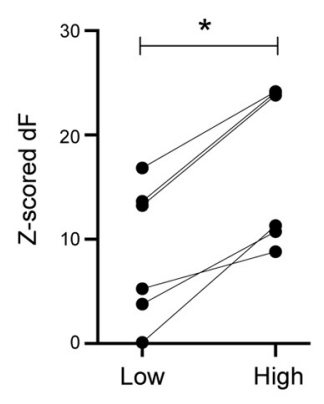

I

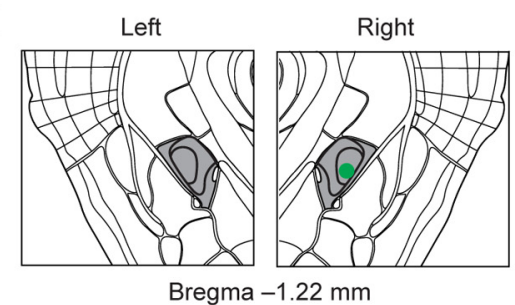

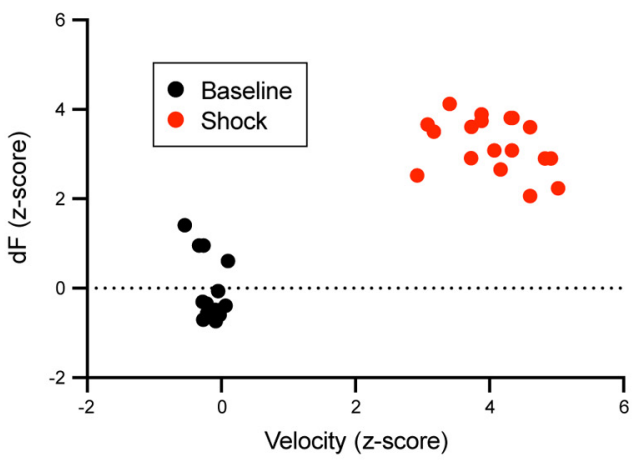

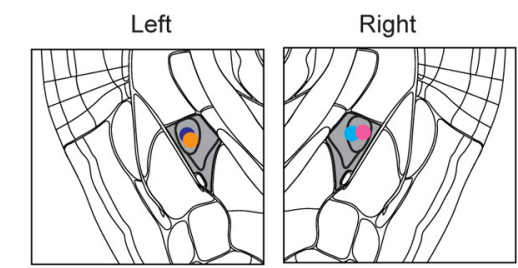

Bregma $-1.34 \mathrm{~mm}$
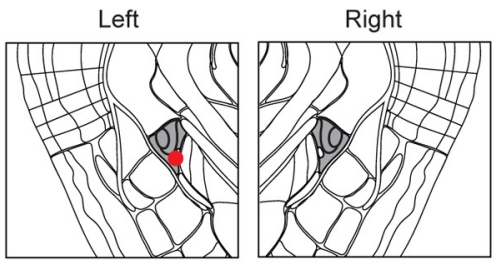

Bregma $-1.46 \mathrm{~mm}$

Figure 4. GPe-projecting CeA neurons encode the information about US during fear conditioning. $\boldsymbol{A}$, Schematic of the approach. $\boldsymbol{B}$, A representative confocal image showing the GPe-projecting CeA neurons expressing GCaMP6f. The track of the implanted optic fiber is also shown. C, Quantification of freezing behavior during retrieval. $\boldsymbol{D}$, Calcium-dependent (solid) and the simultaneously recorded isosbestic (dotted) GCaMP6 fluorescence signals in a representative mouse in $\mathrm{CS}^{+}$and $\mathrm{CS}^{-}$trials for conditioning (left) and retrieval (right) sessions. $\boldsymbol{E}$, Quantification of the calcium-dependent activities in $\mathrm{CS}^{+}$trials during conditioning (left) and retrieval (right; $n=6$ mice). $\boldsymbol{F}$, Relationship between movement velocity and GCaMP6 signals during the shock period or baseline period. $\boldsymbol{G}$, Average responses of GPe-projecting CeA neurons in an example mouse to shocks of low $(0.2$ or $0.4 \mathrm{~mA})$ and high $(0.8$ or $1.0 \mathrm{~mA})$ intensities. $\boldsymbol{H}$, Quantification of responses of all mice $(n=6)$ to shocks of low and high intensities. I, Schematics showing the placement of optic fibers in all the mice used for recording the activities in GPe-projecting (eA neurons with fiber photometry $(n=6$ mice). The CeA is colored in dark gray. Data in $\boldsymbol{C}, \boldsymbol{E}-\boldsymbol{G}$ are presented as mean \pm SEM. ${ }^{*} p<0.05,{ }^{* *} p<0.01,{ }^{* * * *} p<0.0001$. 
A

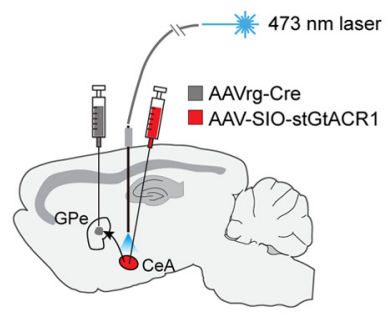

B

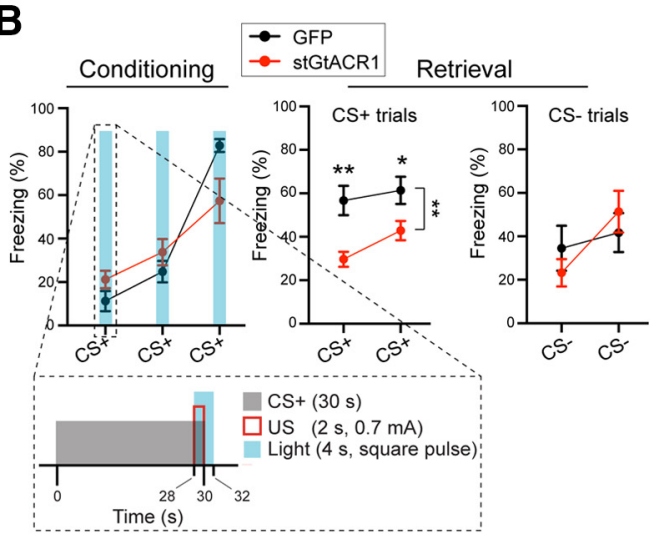

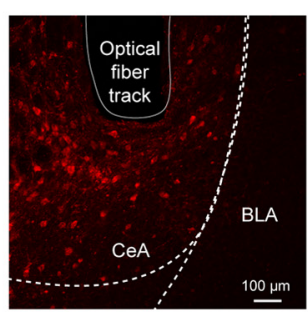

C
D

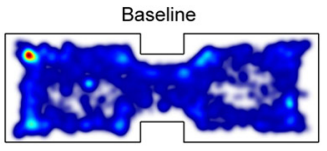

Stimulation in left chamber

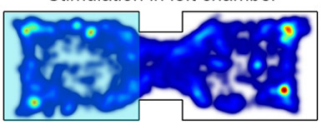

Stimulation in right chamber

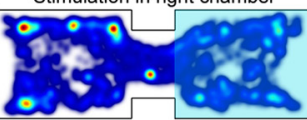

Discrimination

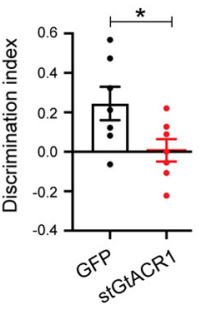

$\mathrm{E}$

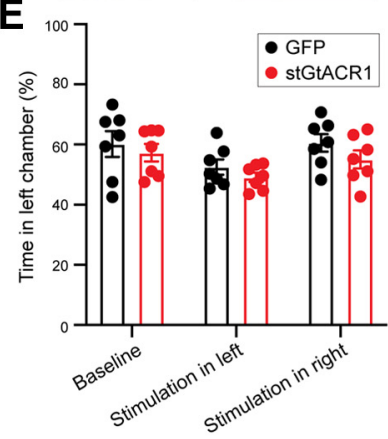

F
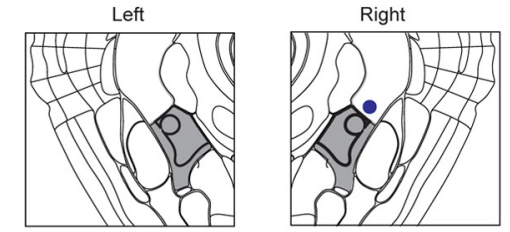

Bregma $-0.94 \mathrm{~mm}$
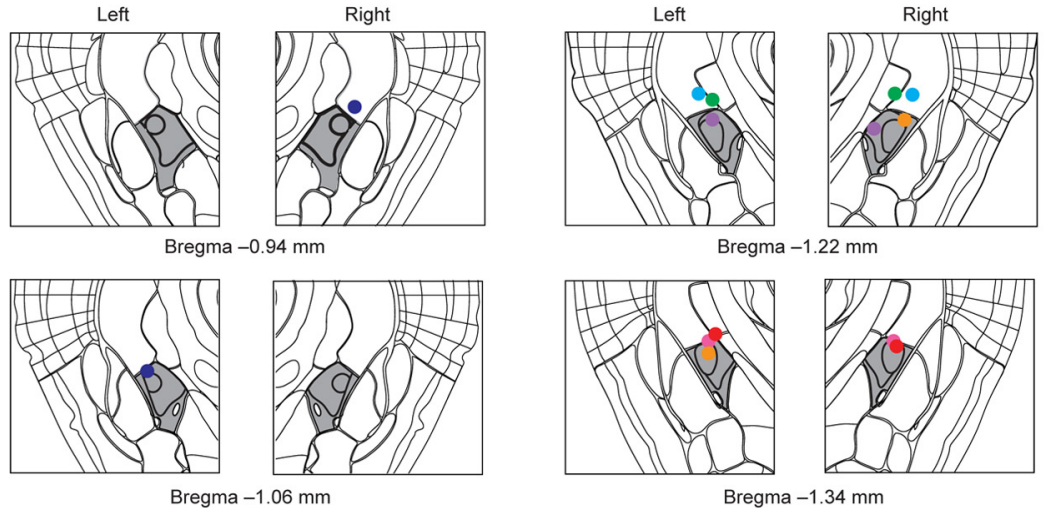

Bregma $-1.22 \mathrm{~mm}$
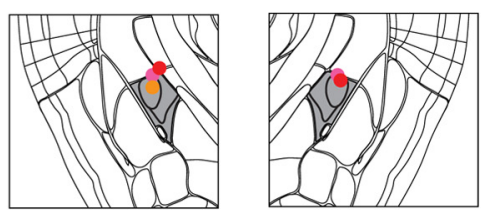

- mouse \#

- mouse \#2

- mouse \#3

- mouse \#4

- mouse \#5

mouse \#6

mouse \#7

Bregma $-1.34 \mathrm{~mm}$

Figure 5. GPe-projecting CeA neuron activity during US presentation is necessary for learning during fear conditioning. $A$, left, Schematic of the approach. Right, Representative confocal image showing the GPe-projecting CeA neurons expressing stGtACR1. The track of the implanted optic fiber is also shown. $\boldsymbol{B}$, Freezing behavior in mice in which GPe-projecting CeA neurons expressed stGtACR1 ( $n=7)$ or GFP $(n=7)$, during conditioning (left) and retrieval (right) sessions. Inset, Structure and timing of CS $^{+}$, US, and light delivery. C, Discrimination Index calculated as [CS ${ }^{+}-$ $\mathrm{CS}^{-} /\left[\mathrm{CS}^{+}+\mathrm{CS}^{-}\right]$, where $\mathrm{CS}^{+}$and $\mathrm{CS}^{-}$represent the average freezing during the presentation of $\mathrm{CS}^{+}$and $\mathrm{CS}^{-}$, respectively. (D) Heat-maps for the activity of a representative mouse at baseline (top), or in a situation whereby entering the left (middle) or right (bottom) side of the chamber triggered photo-inactivation of GPe-projecting CeA neurons. $E$, Quantification of the mouse activity as shown in $\boldsymbol{D}$, for mice in which stGtaCR1 $(n=7)$ or GFP $(n=7)$ was introduced into GPe-projecting CeA neurons. $\boldsymbol{F}$, Schematics showing the placement of optic fibers in all the mice used for inhibiting GPe-projecting (eA neurons with optogenetics ( $n=7$ mice). The CeA is colored in dark gray. Data in $\boldsymbol{B}, \boldsymbol{C}, \boldsymbol{E}$ are presented as mean \pm SEM. ${ }^{*} p<0.05,{ }^{* *} p<0.01$.

$5 A, 6 A-F)$. We subsequently trained the mice in a mild version of the fear conditioning paradigm (Fig. 6B), in which a weak $(0.4 \mathrm{~mA})$ shock was used as the US to avoid the potential ceiling effect a stronger US might have on learning.

During conditioning, three brief trains of photo-stimulation, each coinciding with a US presentation during a trial, were delivered to the CeA (Fig. 6B). This manipulation increased $\mathrm{CS}^{+}$induced conditioned freezing behavior in the ChR2 mice compared with the GFP mice in a retrieval test $24 \mathrm{~h}$ after the conditioning (Fig. 6B). Interestingly, the ChR2 mice also showed an increase in freezing response to $\mathrm{CS}^{-}$during the retrieval test (conditioning: $F_{(1,10)}=3.682, p=0.084$; retrieval, $\mathrm{CS}^{+}$trials: $F_{(1,10)}=5.560,{ }^{\star} p=$ 0.040 ; retrieval, $\mathrm{CS}^{-}$trials: $F_{(1,10)}=16.34,{ }^{* *} p=0.002$; ${ }^{* *} p<0.010$; two-way ANOVA with repeated measures, followed by Sidak's test; Fig. $6 B$ ), albeit their discrimination index did not significantly differ from that of the GFP mice $\left(t_{(7.223)}=1.446, p=0.19\right.$, Welch's $t$ test; Fig. $6 C$ ). To check whether the facilitating effect on learning is because activating GPe-projecting CeA neurons influences valence processing, we tested these mice again in the RTPP or RTPA task for photo-stimulating GPe-projecting CeA neurons using the same parameters as those used in fear conditioning (Fig. 6D). Notably, the two groups of animals behaved similarly in the test $\left(F_{(1,10)}=\right.$ $0.019, p>0.05$; two-way ANOVA with repeated measures; Fig. $6 E$ ), indicating that photo-activation of GPe-projecting CeA neurons is not inherently aversive or rewarding. These results together suggest that activating GPe-projecting CeA neurons during US presentation promotes the formation of fear memories, although the activation may not by itself produce aversive valence.

\section{Discussion}

Animals have the ability to use an environmental cue (i.e., CS) to predict the occurrence of an aversive or harmful consequence (i.e., US), on the condition that the former is frequently associated 
A
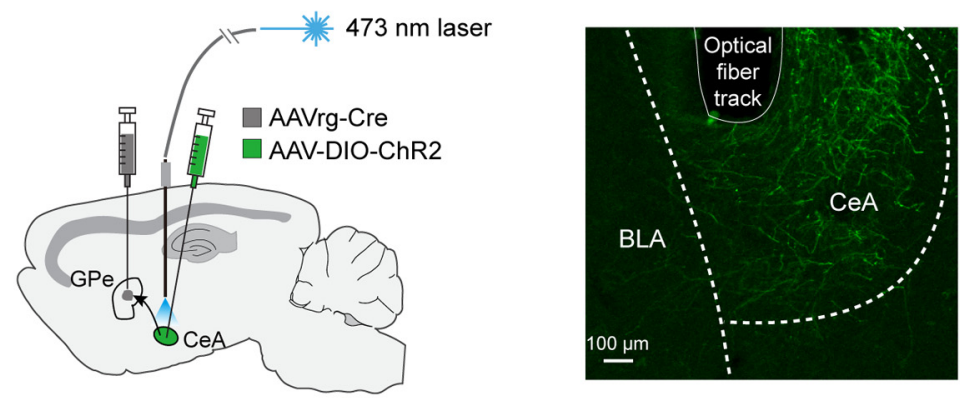

B

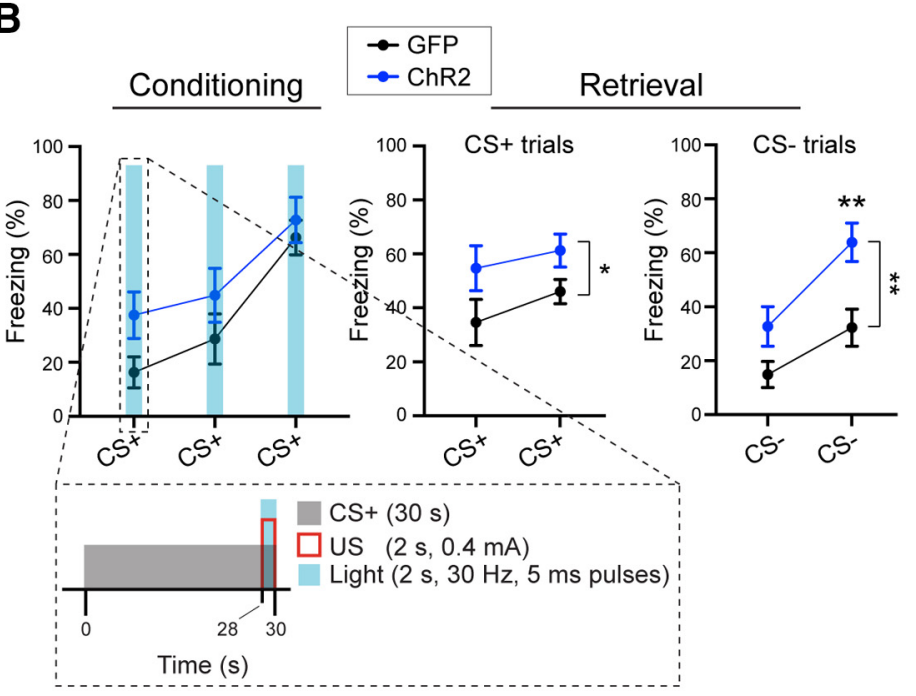

D

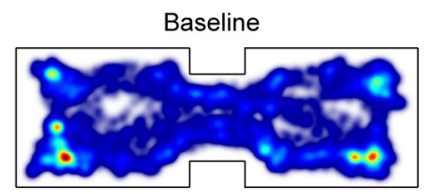

Stimulation in left chamber

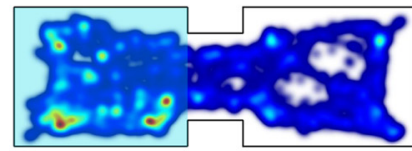

Stimulation in right chamber

\section{Discrimination}

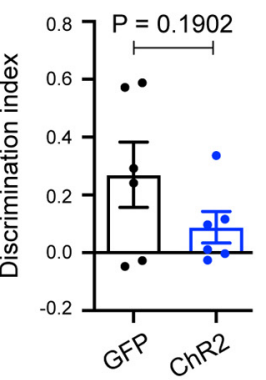

E
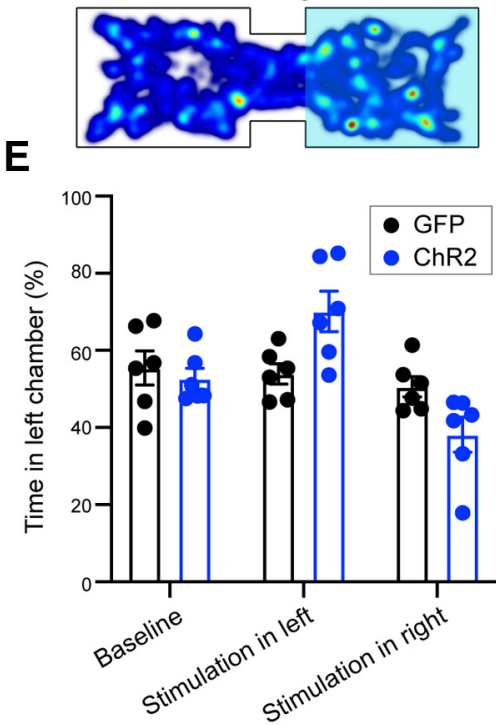

$\mathbf{F}$

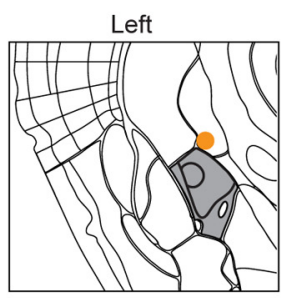

Bregma $-1.06 \mathrm{~mm}$

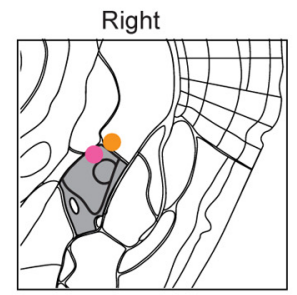

.
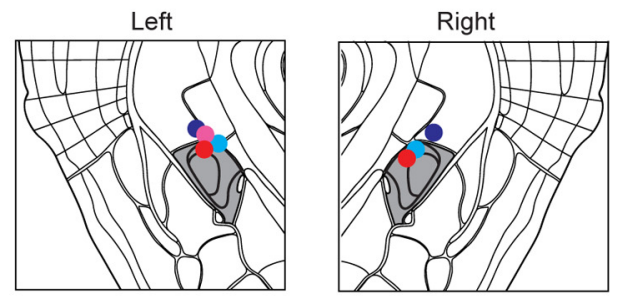

mouse \#1

- mouse \#2

- mouse \#3

- mouse \#4

mouse \#5

Bregma $-1.22 \mathrm{~mm}$

Figure 6. Activation of GPe-projecting CeA neurons during US presentation promotes fear learning. $\boldsymbol{A}$, left, Schematic of the approach. Right, Representative confocal image showing the GPe-projecting CeA neurons expressing ChR2. The track of the implanted optic fiber is also shown. $\boldsymbol{B}$, Freezing behavior in mice in which GPe-projecting (eA neurons expressed ChR2 ( $n=6$ ) or GFP $(n=6)$, during conditioning (left) and retrieval (right) sessions. Inset, Structure and timing of $C^{+}$, US, and light delivery. C, Discrimination Index calculated as $\left[\mathrm{CS}^{+}-\mathrm{CS}^{-} /\left[\mathrm{CS}^{+}+\mathrm{CS}^{-}\right]\right.$, where $\mathrm{CS}^{+}$and $\mathrm{CS}^{-}$represent the average freezing during the presentation of $\mathrm{CS}^{+}$and $C S^{-}$, respectively. $\boldsymbol{D}$, Heat-maps for the activity of a representative mouse at baseline (top), or in a situation whereby entering the left (middle) or right (bottom) side of the chamber triggered photo-activation of GPe-projecting CeA neurons. $\boldsymbol{E}$, Quantification of the mouse activity as shown in $\boldsymbol{D}$, for mice in which ChR2 $(n=6)$ or GFP $(n=6)$ was introduced into GPe-projecting CeA neurons. $\boldsymbol{F}$, Schematics showing the placement of optic fibers in all the mice used for activating GPe-projecting CeA neurons with optogenetics ( $n=5$ mice). The CeA is colored in dark gray. Data in $\boldsymbol{B}, \boldsymbol{C}, \boldsymbol{E}$ are presented as mean \pm SEM. ${ }^{*} p<0.05,{ }^{* *} p<0.01$.

with the latter, and to show appropriate behavioral reactions based on the prediction (Pavlov, 1927; LeDoux, 2000; Lang and Davis, 2006; Schultz, 2006). Such ability is fundamental for survival and adaptation to the environment. Extensive studies, exemplified by those focusing on Pavlovian fear conditioning, have shown that the CeA plays important roles in the establishment of adaptive defensive behaviors (Duvarci and Pare, 2014; Herry and Johansen, 2014; Janak and Tye, 2015; Fadok et al., 2018; Li, 2019). However, despite intensive study, how the CeA processes and represents US information during fear conditioning, and how it contributes to the formation of aversive memories remain to be fully understood. Here, we identified a previously uncharacterized circuit, the CeA-GPe circuit, that is essential for fear conditioning. Specifically, we showed that this circuit predominantly originates from $\mathrm{Sst}^{+} \mathrm{CeA}$ neurons, and permanent inhibition of GPe-projecting CeA neurons prevented fear conditioning. Moreover, GPe-projecting CeA neurons were excited by US but not CS during fear conditioning, and transient inactivation or activation of these neurons specifically during US presentation impaired or promoted, respectively, fear learning. On the basis of these results, we propose that the major function of GPe-projecting CeA neurons in fear conditioning is to process US information, and convey this information to downstream GPe neurons, thereby controlling learning.

A notable observation in our study is that optogenetic activation of GPe-projecting CeA neurons does not induce obvious aversive (or appetitive) responses (Fig. 6D,E). This result is seemingly counterintuitive given the evidence that the CeA-GPe pathway promotes and is critical for fear conditioning, which by 
nature is aversive. There are two possible explanations for this "inconsistency," which are not mutually exclusive. The first possibility is that the CeA-GPe pathway carries only partial information about the US, such as salience, that regulates learning, while parallel pathways originating from the CeA or elsewhere represent valence and other reinforcing properties of the aversive US. Consistent with this scenario, inhibition of GPe-projecting CeA neurons had a relatively small effect on shock responses (Fig. 3D). Such a division of labor has been described for the different projection pathways of the parabrachial nucleus, each of which does not convey the full spectrum of information about US during fear conditioning (Bowen et al., 2020). The second possibility is that the GPe-projecting CeA neurons are functionally heterogeneous, with some neurons representing negative valence and others representing positive valence. As a consequence, activating these neurons indiscriminately produces neither aversive nor appetitive responses. Of note, functional diversity of $\mathrm{SOM}^{+} \mathrm{CeA}$ neurons, which constitute the vast majority of GPe-projecting neurons in the CeA, has been suggested by previous studies (Fadok et al., 2018; Li, 2019). Future studies will disentangle these possibilities.

The GPe is a major basal ganglia structure whose roles in motor control have been the focus of investigation (Kita, 2007; Wallace et al., 2017), but whose other functions have been understudied. Nevertheless, the GPe has been implicated in regulating emotions or affects, including fear or threat. For example, human imaging studies indicate that GPe activation is associated with negative emotions, such as fear, disgust, depression and anxiety (Murphy et al., 2003; Hattingh et al., 2012; Ipser et al., 2013; Binelli et al., 2014). In addition, animal studies have shown that lesions and pharmacological or molecular manipulations in the GPe potently alter fear-like or anxiety-like behaviors (Blanchard et al., 1981; Hernadi et al., 1997; Talalaenko et al., 2006; Kertes et al., 2009; Sztainberg et al., 2011). These findings thus ascribe a function of fear or threat regulation to the GPe. An obvious question is how this GPe function is related to that of the known "fear circuit," including the amygdala. A potential anatomic link between the GPe and the fear circuit is suggested by previous studies, which demonstrate the existence of the CeA to GPe projections (Shinonaga et al., 1992; Hunt et al., 2018). Other inputs to the GPe that convey the affective information of a stimulus, such as the US in fear conditioning, may also exist. Nevertheless, the roles of these projections in fear regulation, and in behavior in general, have remained unknown.

Our study uncovers that the projections from CeA originate mainly from $\mathrm{Sst}^{+}$neurons and shows that the CeA-GPe circuit indeed constitutes a neural substrate for regulating fear learning. The activities of GPe-projecting CeA neurons may not be sufficient to cause aversive responses, as suggested by the observation that activating these neurons produced no effect in the RTPP/ RTPA test. However, the information carried by these neurons could be important for valence processing in the GPe and/or modulating the salience of the US. As it is known that neurons in the sensorimotor and associative striatum both project to the GPe (Tewari et al., 2016), it is possible that the information encoded by GPe-projecting CeA neurons is integrated with that encoded by distinct striatal inputs in GPe neurons to represent the affective properties of the US. Future studies are necessary to elucidate how GPe neurons integrate information from the CeA and the striatum, and interact with neurons in downstream structures to participate in fear processing and learning.

$\mathrm{Sst}^{+} \mathrm{CeA}$ neurons send long-range projections to a number of target areas (Penzo et al., 2014; Yu et al., 2017; Ahrens et al., 2018; Fadok et al., 2018; Zhou et al., 2018; Li, 2019; Ye and
Veinante, 2019; Steinberg et al., 2020). Some of these projections have been studied in the context of fear conditioning or anxietyrelated behaviors (Penzo et al., 2014; Ahrens et al., 2018; Zhou et al., 2018; Steinberg et al., 2020). However, the encoding properties of these projections and how they contribute to specific aspects of learning or executing defensive behaviors have not been characterized. Our study pinpoints that one of the functions of GPe-projecting CeA neurons, which are mainly $\mathrm{Sst}^{+}$, is representation and processing of US-related information during fear conditioning. Future studies need to delineate whether and how different CeA projection pathways differentially but coordinately contribute to the establishment of defensive behaviors.

\section{References}

Ahrens S, Wu MV, Furlan A, Hwang GR, Paik R, Li H, Penzo MA, Tollkuhn J, Li B (2018) A central extended amygdala circuit that modulates anxiety. J Neurosci 38:5567-5583.

Baumann B, Danos P, Krell D, Diekmann S, Leschinger A, Stauch R, Wurthmann C, Bernstein HG, Bogerts B (1999) Reduced volume of limbic system-affiliated basal ganglia in mood disorders: preliminary data from a postmortem study. J Neuropsychiatry Clin Neurosci 11:71-78.

Binelli C, Subirà S, Batalla A, Muñiz A, Sugranyés G, Crippa JA, Farré M, Pérez-Jurado L, Martín-Santos R (2014) Common and distinct neural correlates of facial emotion processing in social anxiety disorder and Williams syndrome: a systematic review and voxel-based meta-analysis of functional resonance imaging studies. Neuropsychologia 64:205-217.

Blanchard DC, Blanchard RJ, Lee MC, Williams G (1981) Taming in the wild Norway rat following lesions in the basal ganglia. Physiol Behav 27:9951000.

Bowen AJ, Chen JY, Huang YW, Baertsch NA, Park S, Palmiter RD (2020) Dissociable control of unconditioned responses and associative fear learning by parabrachial CGRP neurons. Elife 9:e59799.

Cassell MD, Gray TS (1989) The amygdala directly innervates adrenergic (C1) neurons in the ventrolateral medulla in the rat. Neurosci Lett 97:163-168.

Cassell MD, Freedman LJ, Shi C (1999) The intrinsic organization of the central extended amygdala. Ann NY Acad Sci 877:217-241.

Chen TW, Wardill TJ, Sun Y, Pulver SR, Renninger SL, Baohan A, Schreiter ER, Kerr RA, Orger MB, Jayaraman V, Looger LL, Svoboda K, Kim DS (2013) Ultrasensitive fluorescent proteins for imaging neuronal activity. Nature 499:295-300.

Ciocchi S, Herry C, Grenier F, Wolff SB, Letzkus JJ, Vlachos I, Ehrlich I, Sprengel R, Deisseroth K, Stadler MB, Müller C, Lüthi A (2010) Encoding of conditioned fear in central amygdala inhibitory circuits. Nature 468:277-282.

Critchley HD, Melmed RN, Featherstone E, Mathias CJ, Dolan RJ (2001) Brain activity during biofeedback relaxation: a functional neuroimaging investigation. Brain 124:1003-1012.

Davis M (2000) The role of the amygdala in conditioned and unconditioned fear and anxiety. In: The amygdala (Aggleton JP, ed), pp 213-287. Oxford: Oxford University Press.

Duvarci S, Pare D (2014) Amygdala microcircuits controlling learned fear. Neuron 82:966-980.

Duvarci S, Popa D, Paré D (2011) Central amygdala activity during fear conditioning. J Neurosci 31:289-294.

Fadok JP, Krabbe S, Markovic M, Courtin J, Xu C, Massi L, Botta P, Bylund K, Müller C, Kovacevic A, Tovote P, Lüthi A (2017) A competitive inhibitory circuit for selection of active and passive fear responses. Nature 542:96-100

Fadok JP, Markovic M, Tovote P, Lüthi A (2018) New perspectives on central amygdala function. Curr Opin Neurobiol 49:141-147.

García-López M, Abellán A, Legaz I, Rubenstein JL, Puelles L, Medina L (2008) Histogenetic compartments of the mouse centromedial and extended amygdala based on gene expression patterns during development. J Comp Neurol 506:46-74.

Goosens KA, Maren S (2003) Pretraining NMDA receptor blockade in the basolateral complex, but not the central nucleus, of the amygdala prevents savings of conditional fear. Behav Neurosci 117:738-750. 
Govorunova EG, Sineshchekov OA, Janz R, Liu X, Spudich JL (2015) NEUROSCIENCE. Natural light-gated anion channels: a family of microbial rhodopsins for advanced optogenetics. Science 349:647-650.

Hartley ND, Gaulden AD, Báldi R, Winters ND, Salimando GJ, Rosas-Vidal LE, Jameson A, Winder DG, Patel S (2019) Dynamic remodeling of a basolateral-to-central amygdala glutamatergic circuit across fear states. Nat Neurosci 22:2000-2012.

Hattingh CJ, Ipser J, Tromp SA, Syal S, Lochner C, Brooks SJ, Stein DJ (2012) Functional magnetic resonance imaging during emotion recognition in social anxiety disorder: an activation likelihood meta-analysis. Front Hum Neurosci 6:347.

Haubensak W, Kunwar PS, Cai H, Ciocchi S, Wall NR, Ponnusamy R, Biag J, Dong H-W, Deisseroth K, Callaway EM, Fanselow MS, Lüthi A, Anderson DJ (2010) Genetic dissection of an amygdala microcircuit that gates conditioned fear. Nature 468:270-276.

He M, Liu Y, Wang X, Zhang MQ, Hannon GJ, Huang ZJ (2012) Cell-typebased analysis of microRNA profiles in the mouse brain. Neuron 73:35-48.

Hernadi I, Karadi Z, Faludi B, Lenard L (1997) Disturbances of neophobia and taste-aversion learning after bilateral kainate microlesions in the rat pallidum. Behav Neurosci 111:137-146.

Herry C, Johansen JP (2014) Encoding of fear learning and memory in distributed neuronal circuits. Nat Neurosci 17:1644-1654.

Hunt AJ Jr, Dasgupta R, Rajamanickam S, Jiang Z, Beierlein M, Chan CS, Justice NJ (2018) Paraventricular hypothalamic and amygdalar CRF neurons synapse in the external globus pallidus. Brain Struct Funct 223:2685-2698.

Ipser JC, Singh L, Stein DJ (2013) Meta-analysis of functional brain imaging in specific phobia. Psychiatry Clin Neurosci 67:311-322.

Janak PH, Tye KM (2015) From circuits to behaviour in the amygdala. Nature 517:284-292.

Jia H, Rochefort NL, Chen X, Konnerth A (2011) In vivo two-photon imaging of sensory-evoked dendritic calcium signals in cortical neurons. Nat Protoc 6:28-35

Kertes E, László K, Berta B, Lénárd L (2009) Effects of substance P microinjections into the globus pallidus and central nucleus of amygdala on passive avoidance learning in rats. Behav Brain Res 198:397-403.

Keyes PC, Adams EL, Chen Z, Bi L, Nachtrab G, Wang VJ, Tessier-Lavigne M, Zhu Y, Chen X (2020) Orchestrating opiate-associated memories in thalamic circuits. Neuron 107:1113-1123.e4.

Kim CK, Yang SJ, Pichamoorthy N, Young NP, Kauvar I, Jennings JH, Lerner TN, Berndt A, Lee SY, Ramakrishnan C, Davidson TJ, Inoue M, Bito H, Deisseroth K (2016) Simultaneous fast measurement of circuit dynamics at multiple sites across the mammalian brain. Nat Methods 13:325-328.

Kita H (2007) Globus pallidus external segment. Prog Brain Res 160:111-133.

Krettek JE, Price JL (1978) A description of the amygdaloid complex in the rat and cat with observations on intra-amygdaloid axonal connections. J Comp Neurol 178:255-280.

Lang PJ, Davis M (2006) Emotion, motivation, and the brain: reflex foundations in animal and human research. Prog Brain Res 156:3-29.

LeDoux JE (2000) Emotion circuits in the brain. Annu Rev Neurosci 23:155-184.

LeDoux JE, Iwata J, Cicchetti P, Reis DJ (1988) Different projections of the central amygdaloid nucleus mediate autonomic and behavioral correlates of conditioned fear. J Neurosci 8:2517-2529.

Li B (2019) Central amygdala cells for learning and expressing aversive emotional memories. Curr Opin Behav Sci 26:40-45.

Li H, Penzo MA, Taniguchi H, Kopec CD, Huang ZJ, Li B (2013) Experience-dependent modification of a central amygdala fear circuit. Nat Neurosci 16:332-339.

Madisen L, Zwingman TA, Sunkin SM, Oh SW, Zariwala HA, Gu H, Ng LL, Palmiter RD, Hawrylycz MJ, Jones AR, Lein ES, Zeng H (2010) A robust and high-throughput Cre reporting and characterization system for the whole mouse brain. Nat Neurosci 13:133-140.

Mahn M, Gibor L, Patil P, Cohen-Kashi Malina K, Oring S, Printz Y, Levy R, Lampl I, Yizhar O (2018) High-efficiency optogenetic silencing with soma-targeted anion-conducting channelrhodopsins. Nat Commun 9:4125.

Murphy FC, Nimmo-Smith I, Lawrence AD (2003) Functional neuroanatomy of emotions: a meta-analysis. Cogn Affect Behav Neurosci 3:207-233.

Murray AJ, Sauer JF, Riedel G, McClure C, Ansel L, Cheyne L, Bartos M, Wisden W, Wulff P (2011) Parvalbumin-positive CA1 interneurons are required for spatial working but not for reference memory. Nat Neurosci 14:297-299.

Pavlov IP (1927) Conditioned reflexes. London: Oxford University Press.

Penzo MA, Robert V, Li B (2014) Fear conditioning potentiates synaptic transmission onto long-range projection neurons in the lateral subdivision of central amygdala. J Neurosci 34:2432-2437.

Penzo MA, Robert V, Tucciarone J, De Bundel D, Wang M, Van Aelst L, Darvas M, Parada LF, Palmiter RD, He M, Huang ZJ, Li B (2015) The paraventricular thalamus controls a central amygdala fear circuit. Nature 519:455-459.

Schultz W (2006) Behavioral theories and the neurophysiology of reward. Annu Rev Psychol 57:87-115.

Shinonaga Y, Takada M, Mizuno N (1992) Direct projections from the central amygdaloid nucleus to the globus pallidus and substantia nigra in the cat. Neuroscience 51:691-703.

Shucard JL, Cox J, Shucard DW, Fetter H, Chung C, Ramasamy D, Violanti J (2012) Symptoms of posttraumatic stress disorder and exposure to traumatic stressors are related to brain structural volumes and behavioral measures of affective stimulus processing in police officers. Psychiatry Res 204:25-31.

Steinberg EE, Gore F, Heifets BD, Földy MD, Norville ZC, Beier KT, Foldy C, Lerner TN, Luo L, Deisseroth K, Malenka RC (2020) Amygdala-midbrain connections modulate appetitive and aversive learning. Neuron 106:1026-1043.e9.

Swanson LW, Petrovich GD (1998) What is the amygdala? Trends Neurosci 21:323-331.

Sztainberg Y, Kuperman Y, Justice N, Chen A (2011) An anxiolytic role for CRF receptor type 1 in the globus pallidus. J Neurosci 31:17416-17424.

Talalaenko AN, Krivobok GK, Pankrat'ev DV, Goncharenko NV (2006) Neurochemical mechanisms of the dorsal pallidum in the antiaversive effects of anxiolytics in various models of anxiety. Neurosci Behav Physiol 36:749-754.

Taniguchi H, He M, Wu P, Kim S, Paik R, Sugino K, Kvitsiani D, Kvitsani D, Fu Y, Lu J, Lin Y, Miyoshi G, Shima Y, Fishell G, Nelson SB, Huang ZJ (2011) A resource of Cre driver lines for genetic targeting of GABAergic neurons in cerebral cortex. Neuron 71:995-1013.

Tewari A, Jog R, Jog MS (2016) The striatum and subthalamic nucleus as independent and collaborative structures in motor control. Front Syst Neurosci 10:17.

Tovote P, Esposito MS, Botta P, Chaudun F, Fadok JP, Markovic M, Wolff SB, Ramakrishnan C, Fenno L, Deisseroth K, Herry C, Arber S, Lüthi A (2016) Midbrain circuits for defensive behaviour. Nature 534:206-212.

Veening JG, Swanson LW, Sawchenko PE (1984) The organization of projections from the central nucleus of the amygdala to brainstem sites involved in central autonomic regulation: a combined retrograde transport-immunohistochemical study. Brain Res 303:337-357.

Wallace ML, Saunders A, Huang KW, Philson AC, Goldman M, Macosko EZ, McCarroll SA, Sabatini BL (2017) Genetically distinct parallel pathways in the entopeduncular nucleus for limbic and sensorimotor output of the basal ganglia. Neuron 94:138-152.e5.

Waraczynski M (2016) Toward a systems-oriented approach to the role of the extended amygdala in adaptive responding. Neurosci Biobehav Rev 68:177-194.

Wilensky AE, Schafe GE, Kristensen MP, LeDoux JE (2006) Rethinking the fear circuit: the central nucleus of the amygdala is required for the acquisition, consolidation, and expression of Pavlovian fear conditioning. J Neurosci 26:12387-12396.

Ye J, Veinante P (2019) Cell-type specific parallel circuits in the bed nucleus of the stria terminalis and the central nucleus of the amygdala of the mouse. Brain Struct Funct 224:1067-1095.

Yu K, Garcia da Silva P, Albeanu DF, Li B (2016) Central amygdala somatostatin neurons gate passive and active defensive behaviors. J Neurosci 36:6488-6496.

Yu K, Ahrens S, Zhang X, Schiff H, Ramakrishnan C, Fenno L, Deisseroth K, Zhao F, Luo MH, Gong L, He M, Zhou P, Paninski L, Li B (2017) The central amygdala controls learning in the lateral amygdala. Nat Neurosci 20:1680-1685.

Zhou M, Liu Z, Melin MD, Ng YH, Xu W, Südhof TC (2018) A central amygdala to zona incerta projection is required for acquisition and remote recall of conditioned fear memory. Nat Neurosci 21:1515-1519. 